\title{
Integration of IL-2 and IL-4 Signals Coordinates Divergent Regulatory T cell Responses and Drives Therapeutic Efficacy
}

(1)

Julie Y. Zhou ${ }^{1}$, Carlos A. Alvarez ${ }^{1}$, Brian A. Cobb ${ }^{1, *}$

\footnotetext{
${ }^{1}$ Department of Pathology, Case Western Reserve University School of Medicine, Cleveland OH 44106
}

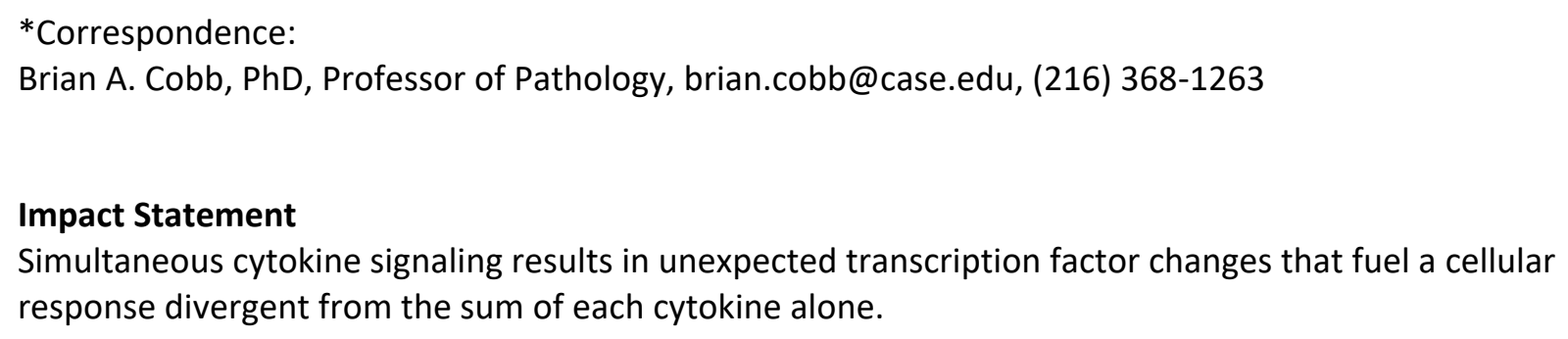

\section{Keywords}

cytokine, T cell, STAT5, STAT6, cytokine receptors, interleukin-2, interleukin-4, interleukin-10, asthma, experimental autoimmune encephalomyelitis 


\section{Abstract}

Cells exist within complex milieus of communicating factors, such as cytokines, that combine to 21 generate context-specific responses, yet nearly all knowledge about the function of each cytokine and 22 the signaling propagated downstream of their recognition is based upon the response to individual 23 cytokines. Here, we found that regulatory T cells (Tregs) integrate concurrent signaling initiated by IL-2 24 and IL-4 to generate a response divergent from the sum of the two pathways in isolation. IL-4 25 stimulation of STAT6 phosphorylation was blocked by IL-2, while IL-2 and IL-4 synergized to enhance 26 STAT5 phosphorylation, IL-10 production, and the selective proliferation of IL-10-producing Tregs, 27 leading to increased inhibition of conventional T cell activation and the reversal of asthma and multiple 28 sclerosis in mice. These data define a mechanism of combinatorial cytokine signaling and lay the 29 foundation upon which to better understand the origins of cytokine pleiotropy while informing 30 improved the clinical use of cytokines. 


\section{$\underline{\text { Introduction }}$}

IL-2 and IL-4 are both pleiotropic cytokines and members of a family in which $\gamma C$ (CD132) is a shared receptor subunit. These and many other cytokines have undergone intensive study because of their ubiquitous and essential roles in health and disease (1), yet their functions can be highly divergent and context dependent.

IL-2 is critical for launching an effector/memory $T$ cell response as a part of normal immune protection. From a pro-inflammatory perspective, IL-2 can drive the proliferation of activated $\mathrm{CD}^{+}{ }^{+}$and CD8 ${ }^{+}$effector T cells $(2,3)$, enhance natural killer cell cytotoxicity $(4,5)$, and augment $B$ cell proliferation and antibody secretion (6). However, IL-2 is also potently anti-inflammatory, driving Fas-mediated activation-induced cell death (7) while promoting Treg survival (8), with its loss resulting in severe autoimmunity and inflammation (9). Its pleiotropy can be partly explained by the strategic and context specific expression of its receptors, with IL-2 receptor $\alpha$ (IL-2R $\alpha$; CD25) being a common marker for activated conventional T cells (Tconv) as well as an established constitutive marker for Tregs $(10,11)$. CD25 is part of the high affinity receptor complex with IL-2 receptor $\beta$ (IL-2R 3 ; CD122) and $\gamma C$, although it can function as a low-affinity receptor in isolation (12). IL-2 activity is dependent upon Jak/STAT signaling, with STAT3 and STAT5 being the primary mediators of its transcriptional regulation of target genes (13).

IL-4 function has perhaps an even broader impact and has two receptor forms. The type 1 IL-4 receptor is comprised of IL-4 receptor $\alpha$ (IL-4R $\alpha$; CD124) and $\gamma \mathrm{C}$, whereas the type II receptor contains IL-4R $\alpha$ and IL-13 receptor $\alpha 1$ (IL-13R $\alpha$; CD213A1)(14). Like many cytokines, signaling is mediated by the Jak/STAT pathway, with STAT6 being a main transcriptional mediator (15) (16). IL-4 is historically associated with allergy through its ability to stimulate class switching to IgE and expression of the IgE receptor (17), although it is also critical for parasite clearance (18) and differentiation of naïve T cells into a type 2 helper (Th2) phenotype (19), all of which is considered pro-inflammatory. Yet, IL-4 is also a key differentiating factor for wound healing macrophages (20), which is essential for tissue repair, and is known to oppose IL-17-mediated inflammatory diseases like psoriasis and experimental autoimmune encephalomyelitis (EAE) $(21,22)$. In fact, IL-4 has been shown to elicit IL-10 in both macrophages and T cells $(23,24)$, helping to explain how IL-4 is also potently anti-inflammatory.

Although much is known about the signaling initiated by these and most other cytokines, understanding of how their signals integrate in complex environments remains limited. For many cytokines, the JAK/STAT cascades underlie their transcriptional impact (25), although a small number of JAK and STAT proteins belies the divergent nature of the responses. STAT homo- and heteromultimerization is one mechanism that exponentially expands the available transcription outcomes (26). There is also evidence that cytokines can lead to the transcriptional enhancement or diminution of the signaling machinery for other cytokines (27). For example, IL-2 stimulation drives the expression of IL$4 R \alpha$, thereby enhancing subsequent responses to IL-4 through serial activation of STAT5 and STAT6, respectively, ultimately leading to Th2 T cell differentiation ((28)). This is additive signaling, whereby the canonical pathways act in concert to drive $T$ cell differentiation, and follows our knowledge of each pathway in isolation. These mechanisms partially explain how a small number of STAT molecules can drive diverse cellular outputs, but do not account for the possibility of simultaneous stimulation that may occur in the complex array of cytokines that exist in any biological environment.

Here, we have discovered that the combination of IL-2 and IL-4 stimulation in Tregs culminates in a synergistic enhancement of Treg function and proliferation in vitro and in vivo. This effect is driven by a uniquely integrated response requiring simultaneous rather than serial stimulation. These findings reveal a novel mechanism underlying cytokine signaling which can drive unexpected outcomes that are differential to the simple combination of pathways, thereby laying the groundwork for a more complete 
picture of cytokine pleiotropy and potentially leading to enhancements to the use of cytokines in the clinical setting.

\section{$\underline{\text { Results }}$}

\section{IL-2 and IL-4 synergistically promote IL-10 production by Tregs}

Our previous findings demonstrated that IL-4 impacts IL-10 production in FoxP3 ${ }^{+}$Tregs (29), but the nature of the functional interaction between IL-4 and IL-2, a key survival factor for Tregs in vitro and in vivo, remained obscure. Thus, we created a novel dual reporter mouse by crossing FoxP3 ${ }^{\mathrm{RFP}}(30)$ and IL$10^{\mathrm{GFP}}(31)$ mice, thereby enabling live sorting of FoxP3 ${ }^{+}$cells and analysis of IL-10 production on a per-cell basis. $\mathrm{CD}^{+} \mathrm{FoxP3}^{+}$Tregs isolated from the spleens of naïve dual reporter mice (Figure 1 - figure supplement $1 \mathrm{~A}-\mathrm{B}$ ) by magnetic bead and sterile fluorescence-activated cell sorting (FACS) were cultured with T cell receptor (TCR) activation using $\alpha \mathrm{CD} 3 \varepsilon$ antibody and all combinations of IL-2 and IL-4 for 3 days. We found that Tregs cultured with combinatorial cytokine stimulation resulted in synergistically higher numbers of IL-10 expressing cells (Figures 1A-1C) and IL-10 secretion (Figure 1D) compared to single cytokine stimulation. However, analysis of $\mathrm{IL}-10^{+}$cells revealed that $\mathrm{IL}-10$ expression was no different than IL-2-stimulation in isolation (Figure $1 \mathrm{E}$ ), suggesting that the cytokines in combination do not elicit a synergistic increase in IL-10 production on a per-cell basis. The sex-independent (Figure $1 \mathrm{~F}$ ) and TCR-stimulation dependent synergy (Figures 1A-1D) was present in FoxP3 ${ }^{+}$Tregs but not FoxP3 Tconv (Figure $1 \mathrm{~A}$ ), and no loss of FoxP3 expression was observed (Figure 1G), suggesting that the machinery required for this effect was unique to Fox $3^{+}$Tregs.

In order to determine whether the cytokines must be present at the same time, we isolated Tregs and stimulated them with cytokines in series over a 3-day culture and compared the response to simultaneous stimulation. We discovered that both IL-2 and IL-4 must be present in culture together to manifest the synergistic IL-10 response (Figures $1 \mathrm{H}-\mathrm{I}$ and Figure 1 - figure supplement $3 \mathrm{~A}-\mathrm{B}$ ), implicating the integration of IL-2 and IL-4-mediated intracellular signaling as a key underlying mechanism.

To ascertain whether IL-2/IL-4 also affects the ability of Tregs to produce other cytokines and chemokines, Treg supernatants after 3 days of culture with or without TCR stimulation and all cytokine combinations were analyzed by multianalyte Luminex. Remarkably, no cytokine or chemokine other than IL-10 was synergistically and robustly increased (Figure 1 - figure supplement 2 ) by combined IL-2 and IL-4, including the Treg-associated TGF- $\beta$ family members (32)(Figure 1J). Moreover, neither IL-2 nor IL-4 induced autocrine production of the reciprocal cytokine (Figure 1K).

To differentiate whether the combined cytokines induced or maintained IL-10 expression in Tregs, we sorted splenocytes from the dual reporter mice into FoxP3 ${ }^{+} \mathrm{IL}-10^{-}$and FoxP3 ${ }^{+} \mathrm{IL}-10^{+}$populations, then cultured them in all combinations. We observed robust induction of IL-10 expression in Fox $3^{+} \mathrm{IL}-10^{-}$cells and maintenance of IL-10 expression in FoxP3 ${ }^{+} \mathrm{IL}-10^{+}$cells, with no detectable change in FoxP3 (Figures $1 \mathrm{~L}$ and Figure 1 - figure supplement $3 \mathrm{C}$ ). To assess this effect beyond 3 days, we cultured Tregs and analyzed them on days 3-7 and found a profound daily increase in the overall percentage of IL-10 ${ }^{+}$Tregs, ultimately leading to nearly every Treg converting to IL-10 $0^{+}$by day 7 (Figures $1 \mathrm{M}$ and Figure 1 - figure supplement 3D).

\section{IL-2/IL-4 enhances Treg proliferation and selectively drives the expansion of IL-10 ${ }^{+}$Tregs}

The lack of a major difference in per-cell IL-10 expression after exposure to IL-2 and IL-4 together (Figure 1E) coupled with the conversion of nearly all Tregs into IL-10+ cells (Figure $1 \mathrm{M}$ ) and synergistic IL10 release (Figure 1D) suggested that the cells were proliferating in response to the combined cytokines. Dual reporter Tregs were isolated and stained with CellTrace Violet and stimulated with all cytokine combinations for 3 days. Tregs cultured with the combined cytokines were more proliferative, as measured by the number and magnitude of cell division peaks (Figure 2A), proliferation index, and 
division index (Figure 2B). The division index revealed that more Tregs stimulated by the combined cytokines divide, and the proliferation index indicated that each dividing Treg undergoes more divisions than when stimulated with or without the single cytokines.

Since IL-2 and IL-4 in combination elicited synergistic IL-10 production by Tregs (Figure 1), we determined whether Treg proliferation and viability was correlated with higher IL-10 expression among dividing cells. We found that cytokine supplementation led to each subsequent generation of Tregs expressing more IL-10 than the previous generation (Figure $2 \mathrm{C}$ ). Furthermore, by staining IL-10 ${ }^{-}$and IL$10^{+}$Tregs with CellTrace prior to culturing and Sytox viability dye after culturing revealed that originally IL-10- Tregs that gained IL-10 expression adopted a higher division index than the Tregs which remained IL-10- (Figure 2D), and Tregs that were IL- $10^{+}$on day 0 and remained IL-10 $10^{+}$after 3 days of culture had the highest division index and percentage of the overall population that divided. Lastly, the IL-10 $10^{+}$Tregs that lost IL-10 expression had a drastic decrease in proliferation as measured by division index and percentage of cells that underwent division, indicating that IL-10 $0^{+}$Tregs only lose IL-10 expression when they become quiescent and non-dividing.

We then compared the division and proliferation indices of $\mathrm{IL}-10^{+}$with IL-10- Tregs, and found that IL-10-expressing Tregs were indeed dramatically more proliferative than Tregs that do not express IL-10 (Figure 2E), and that IL-10+ Tregs supplemented with combined IL-2 and IL-4 proliferated the most of all. Moreover, the increased proliferation and division indices in IL-2/IL-4-treated IL-10 ${ }^{+}$Tregs compared to IL-10- Tregs suggests that expression of IL-10 in Tregs, as induced by the combined cytokines, coincides with more cell proliferation (Figure $2 \mathrm{~F}$ ).

Finally, in a time course study where purified Tregs were cultured in the presence of all cytokine combinations for 3-7 days, the combined IL-2 and IL-4 synergistically increased the number of IL-10 ${ }^{+}$ Tregs compared to single cytokines, most notably increasing the counts of IL-10 $0^{+}$Tregs over 12 -fold in 5 days (Figures $2 \mathrm{G}$ ). As seen with IL-10 induction (Figure $1 \mathrm{H}$ ), exposing cells to the cytokines in series rather than together failed to duplicate the proliferative response (Figure $2 \mathrm{H}$ ).

Collectively, these data (Figures 1-2 and Figure 1 - figure supplements 1-3) indicate that not only does the combination of IL-2 and IL-4 lead to strong IL-10 expression and enhanced proliferation among TCR-activated Tregs, but that these events are associated in such a way that leads to the selective propagation of IL-10 ${ }^{+}$Tregs resulting in an exponentially increased Treg response.

\section{Combined IL-2 and IL-4 increase the suppressive ability of Tregs}

The ability of IL-10 to suppress the proliferation and functions of conventional and effector T cells is well known (33). Since the cytokines working together elicit extraordinarily robust IL-10 production by Tregs through a combination of IL-10 induction and cellular proliferation, we assessed the in vitro suppressive impact on Tconv proliferation and cytokine production. Tregs were stimulated as before for 3 days, washed, counted, and normalized to the number of surviving Tregs, and placed them in coculture with freshly purified and CellTrace-stained Tconv cells harvested from the spleens of dual reporter mice. After 3 days, the cells were analyzed for proliferation and cytokine production. Treg suppression activity was impaired upon IL-4 stimulation, despite the IL-4-mediated increase in IL-10 (Figure 1), while supplementation with either IL-2 or both cytokines substantially increased Tconv inhibition (Figure 3A). Stimulation by combined IL-2 and IL-4 led to increased IL-10 release by Tregs (Figures 3B) and significant suppression of IL-4 and IL-17A production by Tconv cells (Figures 3C), but only a modest increase in suppressive capacity over IL-2 exposure alone.

Next, to assess the role of IL-10 in Tconv inhibition, we repeated the experiment using Tregs isolated from IL-10-deficient (IL-10 $1-$ ) mice and found that cytokine-stimulated IL-10 ${ }^{--}$Tregs failed to suppress Tconv proliferation (Figure 3D), did not express IL-10 (Figures 3E), and failed to reduce Tconv cytokine expression (Figures 3F). This demonstrates that IL-10 is necessary for cytokine-stimulated Tregs to suppress Tconv cells. 
These data show that on a per-cell basis, Tregs stimulated with IL-2 alone have similar suppressive activity compared to the IL-2/IL-4 combination, and that this activity is IL-10 dependent. This agrees with the lack of difference in per-cell IL-10 production (Figure 1E), but fails to account for the enhanced and selective proliferation of $\mathrm{IL}-10^{+}$Tregs with combinatorial cytokine exposure. To do this, we cultured Tregs alone with all combinations of cytokines for 7 days (Figures 3A-3C) and washed them after stimulation, but did not re-normalize the cell numbers prior to co-culture with fresh Tconv cells to allow for proliferative differences to be incorporated into the activity assessment. We observed that Tregs cultured without cytokine had lost their suppressive ability, likely due to an extended amount of time without supportive growth signals in culture, and that the Tregs cultured with single cytokines maintained suppressive activity (Figures 3G-3I). However, Tregs stimulated with both cytokines showed significantly greater suppression of Tconv proliferation (Figure 3G), produced higher concentrations of IL-10 (Figures $3 \mathrm{H}$ ), and suppressed the production of IL-4 and IL-17A by Tconv cells to a greater degree (Figures 3I) compared to single cytokine conditions. Indeed, we found that by day 9 of a 1:8 Treg-Tconv co-culture, the Tregs previously stimulated with both IL-2 and IL-4 comprised over $80 \%$ of the resulting culture (Figure 3J), demonstrating that the synergistic impact of these cytokines is manifested through the combination of IL-10 production, suppression of Tconv proliferation and cytokine production, and the selective proliferation of $\mathrm{IL}-10^{+}$Tregs even after removal from recombinant cytokine stimulation.

\section{Synergistic IL-10 production and proliferation is STAT5-dependent}

The intracellular signaling downstream of IL-2 and IL-4 receptors is well known, including STAT6 as characteristic of IL-4 signaling (34) and STAT5 being associated with IL-10 production in Tregs downstream of IL-2 (35). In order to understand the mechanism by which IL-2 and IL-4 signals are integrated, we measured STAT phosphorylation by flow cytometry following Treg stimulation as before. While STAT3 is associated with both IL-2 and IL-4, we found that STAT3 phosphorylation was not altered as a result of combined cytokine stimulation in TCR-activated Tregs, although a small amount of STAT3 phosphorylation occurred at 60 minutes with combined cytokines without TCR stimulation (Figure 4A). IL-2 induced low levels of STAT6 phosphorylation, while IL-4 induced robust STAT6 phosphorylation in TCR-activated Tregs. Remarkably, the addition of IL-2 to the IL-4 and TCR signals resulted in the reversal of IL-4-dependent STAT6 activation (Figure 4B). Mirroring IL-10 production, we also detected a dramatic and synergistic increase in STAT5 phosphorylation when TCR-activated Tregs were given both cytokines (Figure 4C), suggesting that STAT5 may be responsible for the synergistic IL-10 and proliferative response.

In order to determine whether STAT5 activation is a point of signaling convergence necessary for synergy, we blocked STAT5 activity over a range of increasing inhibitor (STAT5i) doses in stimulated Tregs. Blockade of STAT5 activity inhibited the response by over $80 \%$ in a dose-dependent fashion, as measured by inhibition of IL-10 induction as a percent (Figure 4D) and number (Figure 4E) of IL-10 ${ }^{+}$ Tregs, overall Treg viability (Figure 4 - figure supplement 1A), IL-10 expression on a cellular level (Figure 4 - figure supplement 1B), proliferation (Figure 4 - figure supplement 4C-D), and IL-10 release (Figure 4 - figure supplement 1E). As a control, analysis of IFNY, a cytokine not produced synergistically following combined IL-2 and IL-4 stimulation, demonstrated no inhibition (Figure 4 - figure supplement 1F).

These data demonstrated that IL-10 production and proliferation downstream of IL-2 and IL-4 was dependent upon STAT5; however, it remained unclear whether cytokine synergy also required STAT5. We again cultured Tregs with the single or combinatorial cytokines and a range of increasing concentrations of STAT5i. Although STAT5i inhibited IL-10 expression and reduced the number of IL-10 ${ }^{+}$ Tregs under all conditions (Figure 4F-4G), we discovered that at high STAT5i concentration, IL-2/IL-4 synergy is lost, as illustrated by a lack of difference between the sum of the individual IL-2 and IL-4 responses (Figures 4F-4G; stacked bars) and stimulation with both cytokines simultaneously (Figures 4F4G; purple bars). 
These data demonstrate that the signaling downstream of individual cytokine receptors can be altered by the combinatorial integration of other cytokine-stimulated signals, as is clear for STAT5 and STAT6. Moreover, it is clear that not only are IL-10 production and Treg proliferation paired events, but that STAT5 is necessary to induce the synergistic response characteristic of the IL-2 and IL-4 combination.

\section{Combinatorial IL-2 and IL-4 signaling promotes the expression of the Type 2 IL-4 receptor}

We have found that IL-2 and IL-4 in combination promotes selective proliferation of IL-10 ${ }^{+}$Tregs (Figure 2), which suggests that the receptor expression may change over time to promote the maintenance of IL-10 expression. Thus, we measured cell surface expression of all IL-2 and IL-4 receptor subunits. Tregs were again stimulated, and each subunit was quantified by flow cytometry (Figure 5). Surface expression was first visualized using principle component analysis (PCA), which demonstrates that the receptor expression pattern of TCR-activated Tregs without cytokines, with IL-2 or IL-4 individually, and both cytokines are all divergent and distinct (Figure 5A), further supporting a model in which Tregs possess the ability to integrate multiple cytokine signals in ways distinct from simply adding known pathways studied in isolation. Further analyses of the data revealed a strong positive correlation between IL-10 and IL-2R $($ CD25) expression (Figure 5B), which agrees with both a prior study which reported STAT5-dependent expression of IL-2R $\alpha$ on Tregs (36) and our STAT5 data (Figure 4C). We also discovered a strong negative correlation between IL-10 and IL-2R $\beta$ expression (Figure 5C), and although there is a positive correlation between IL-10 and $\gamma C$ chain in cells receiving individual cytokines, this is significantly reduced when IL-2 and IL-4 are together (Figure 5D). Looking at the IL-4 receptor subunits, the IL-4R $\alpha$ expression pattern (Figure 5E) mirrors that observed for the $\gamma C$ chain (Figure 5D), with a positive correlation with IL-10 for the individual cytokines, but less so with the combination. Curiously, IL-13R $\alpha 1$ expression showed a strong positive correlation with IL-10 (Figure 5F), similar to IL-2R $\alpha$ (Figure $5 B$ ), following combined cytokine stimulation. Since the type II IL-4R is used by both IL-4 and IL-13, we tested whether IL-13 could achieve the same synergistic Treg outcome as IL-4 in combination with IL-2. Supplementing Treg cultures with recombinant IL-13 in any combination with IL-2 and IL-4 did not impact IL-10 production (Figures $5 \mathrm{G}-\mathrm{H}$ ), suggesting that the response is specific for IL-4. These data collectively demonstrate that IL-2R $\alpha$ and the type II IL-4R (IL-4R $\alpha+$ IL-13R $\alpha$ ) are selectively associated with the synergistic response to the IL-2 and IL-4 combination.

\section{Combinatorial IL-2 and IL-4 signaling suppresses asthma-like pulmonary morbidity}

Given the potent in vitro Treg response to the combination of IL-2 and IL-4 and the resulting integration of their signaling pathways, we next sought to determine the in vivo response within the context of inflammatory disease. Since this pathway was implicated using murine asthma (29, 37-39), we first challenged mice with house dust mite (HDM) over the course of 2 weeks to induce pulmonary inflammation as described previously (29). The combinatorial cytokines were administered i.n. at 3 time points prior to HDM challenge as a prophylactic therapy (Figure 6 - figure supplement $1 \mathrm{~A}$ ). We found that mice pretreated with a 1:10 ratio of IL-2:IL-4 were significantly protected from developing airway disease as quantified by leukocyte airway infiltration in bronchial alveolar lavage fluid (BALf)(Figure 6A). Tissue histology by H\&E staining further demonstrated a reduction of cellular infiltration around the major airways and vasculature, and decreased bronchial epithelial cell hyperplasia (Figure 6B), while PAS staining revealed reduced mucus production (Figure 6C). Flow cytometry of lung and spleen cells showed that the mice administered combined cytokines accumulated more IL- $10^{+}$and total Tregs per gram of tissue (Figure 6D). The cytokines were not able to reduce disease morbidity in mice deficient in IL-10 as indicated by BALf cell differentials and lung histology (Figure 6 - figure supplement 1B). Indeed, IL-10 deficient mice appeared to trend towards more severe disease with cytokine administration. 
We further investigated the efficacy of the combined cytokines as therapy to ongoing disease rather than as a prophylaxis (Figure 6 - figure supplement $1 \mathrm{C}$ ). We found that a 1:1 ratio of IL-2/IL-4 was sufficient to suppress disease, as indicated by reduced BALf cell differentials (Figure 6F) and greatly improved lung histology (Figure 6 - figure supplement 1D). In comparison, individual cytokines administered at the same doses failed to significantly suppress infiltration (Figure 6F) or tissue pathology (Figure 6 - figure supplement 1D).

Since asthma is often associated with IL-4 and type 2 immunity, we used the therapeutic treatment regimen (Figure 6 - figure supplement $1 \mathrm{C}$ ) in the classically Th2-skewed BALB/c mouse background. Remarkably, IL-2 and IL-4 administration in combination significantly suppressed disease when given at the equimolar dose (Figures $6 \mathrm{G}$ and Figure 6 - figure supplement $1 \mathrm{E}$ ), demonstrating that these cytokines do not exacerbate asthma pathology even in a Th2-skewed system.

Finally, asthma in humans often presents as a chronic disease that affects patients for the duration of their life. We therefore tested the efficacy of the cytokines in a chronic model of pulmonary inflammation induced by challenging mice with three alternating antigens (i.e. HDM, cockroach antigen, and ovalbumin) over 10 weeks (Figure 6 - figure supplement 2A). Since this model results in tissue remodeling and hyper-responsiveness to bronchial restricting agents, we examined the lung function of the mice using a Flexivent ventilator, and observed that the mice treated with the combined cytokines had significantly lower lung resistance upon methacholine challenge (Figure 6H). Moreover, lung histology revealed reduced cellular infiltration and epithelial hyperplasia by H\&E staining, decreased mucus production (PAS staining), and prevention of long-term tissue remodeling (TriChrome staining) when provided IL-2 and IL-4 combination therapy (Figure 6 - figure supplement 2B).

These data reveal that the combination of IL-2 and IL-4 serves as a robust preventative and therapeutic treatment for multiple models of murine asthma on both Th1 and Th2-skewed backgrounds.

\section{Combinatorial IL-2 and IL-4 signaling suppresses EAE morbidity}

Since the asthma models allow for direct administration of cytokine to the site of inflammation via inhalation, we sought to determine whether systemic administration of both cytokines would also be beneficial in the IL-17-driven model EAE. EAE was induced in dual reporter mice by sensitization with myelin oligodendrocyte glycoprotein $(\mathrm{MOG})_{35-55}$ peptide and adjuvant. When the combined cytokines were injected i.v. into mice challenged with MOG, all three delivery schedules (Figure 7 - figure supplement $1 \mathrm{~A}$ ), including preventative (i.e. prophylactic), concomitant (i.e. at the first sign of disease), and therapeutic (i.e. approaching peak disease), generated significant reductions in clinical score (Figures 7A and Figure 7 - figure supplement 1B). Histological analyses indicated that mice treated with the combined cytokines had reduced cellular infiltration (Figures $7 \mathrm{~B}$ and Figure 7 - figure supplement 1C; H\&E) and demyelination (Figures $7 B$ and Figure 7 - figure supplement $1 C$; Luxol Fast Blue) of the spinal cord. Flow cytometry further revealed that the cytokines led to significantly reduced MOG-specific Tconv cells (Figure 7C) while ELISA showed decreased IL-17 within neuronal tissues (Figure 7D).

To better understand the underlying mechanism of EAE suppression, we analyzed Tregs within the central nervous system (CNS) and spleen. We found that the cytokines in combination increased the percentage of CNS and splenic IL-10+ Tregs in MOG-challenged animals (Figures 7E and 7F) in correlation with reduced disease. Interestingly, we did not observe increases in IL-10 among CNS-localized F4/80 macrophages in MOG-challenged mice with cytokine therapy (Figure 7G) despite the reported ability of IL-4 alone to induce IL-10 production in macrophages (40). Finally, we found that cytokine efficacy in EAE was lost in IL-10-deficient mice as measured by disease score (Figure 7H), cellular infiltration by H\&E (Figure 7I) and IL-17A levels in the CNS (Figure 7J), thereby demonstrating the IL-10-dependency of combined IL-2 and IL-4 in suppressing EAE. 
bioRxiv preprint doi: https://doi.org/10.1101/2020.04.14.040485; this version posted April 14, 2020. The copyright holder for this preprint (which was not certified by peer review) is the author/funder, who has granted bioRxiv a license to display the preprint in perpetuity. It is made available under aCC-BY 4.0 International license. 


\section{Discussion}

In this study, we discovered that the combinatorial signaling mediated by IL-2 and IL-4 in TCRactivated Tregs produced a response characterized by IL-10 expression synergy, selective proliferation of IL- $10^{+}$Tregs, differential STAT5 and STAT6 signaling, and distinct cell surface receptor phenotype compared to either cytokine in isolation. These factors cumulatively enhanced the ability of Tregs to suppress Tconv cell proliferation and cytokine secretion in co-culture while successfully attenuating inflammatory diseases of different underlying etiologies in preventative, concomitant, and therapeutic delivery regimens. Disease suppression was paired with increased Treg prevalence and IL-10 expression in Tregs, a reduction in MOG-specific effector T cells in EAE, and was abrogated in mice deficient in IL10. Together, our data reveal that the combinatorial cytokine signaling in Tregs is integrated to generate a unique biological outcome that is divergent from the sum of each pathway in isolation and robustly enhances and maintains the suppressive ability of $\mathrm{FoxP}^{+}$Tregs in vitro and in vivo.

Previous studies have shown that two cytokines can cooperate by sharing the same STAT proteins to either enhance or interfere with the other to form a different outcome $(27,41,42)$. While our study focuses on Tregs, others have reported optimal differentiation of Th2 cells with sequential IL- 2 and then IL-4 stimulation, which upregulates IL-4R $\alpha$ and is mediated by combined STAT5 and STAT6 phosphorylation $(43,44)$. Different from the sequential nature of these and other studies (27), our principle findings required simultaneous stimulation by both cytokines (Figure $1 \mathrm{H}-\mathrm{I}$ ). Furthermore, while FoxP3- Tconv cells were reported to undergo a Th2-differentiating response to IL-4 and IL-2 (43), we found that this combination failed to induce IL-10 production in FoxP3- Tconv cells (Figure 1A) while programming Fox $3^{+}$Tregs towards an $\mathrm{IL}^{-10^{+}}$and suppressive phenotype. As a result, our data reveal that not only can the integration of multiple cytokine pathways diverge from the sum of the parts, but that the combinatorial nature can be different among varied cell types and lineages.

Interestingly, our data indicate that administration of IL-2 and IL-4 in mice in vivo suppressed the severity of not only IL-17-dependent EAE (45), but also several models of murine asthma in both Th1 and Th2-skewed backgrounds (46). This initially appears at odds with the notion that administration of IL-4 in asthmatic mice aggravates disease (47). However, we found that the combination of IL-2 and IL-4 develops Tregs with highly effective suppression of IL-4 production in Tconv cells in vitro (Figure 3). With the loss of IL-4-driven STAT6 phosphorylation in Tregs stimulated both cytokines, we have demonstrated that the IL-4 signal leading to STAT6 activation is dramatically inhibited upon simultaneous IL-2 exposure in Tregs. This reinforces the notion that the functional outcome of a cytokine is highly context dependent and may not reflect the outcome of the cytokine in isolation, leading to pleiotropy in complex systems.

In the case of IL-2 and IL-4, the net functional result of the discovered pathway is a greatly enhanced population of immune inhibitory IL-10 $10^{+}$Tregs. The cellular activity was potent enough to not only reduce disease prophylactically, but also to reverse ongoing inflammation in both asthma and EAE models. It is therefore interesting to note that the use of both IL-2 and IL-4 appears to be a characteristic of some beneficial microbiota antigen responses. For example, Bacteroides fragilis, which is native to a healthy human gut $(48,49)$, is known to regulate the peripheral immune system $(37,38,50,51)$ and even benefit the gut-brain axis $(52,53)$ through $T$ cell recognition of its capsular polysaccharide PSA $(54)$. It is now clear that PSA-specific effector T cells require communication with Tregs, and that immune suppression and inflammatory disease reversal depends upon both IL-4 $(29,39)$ and IL-2 $(55)$, thereby providing a biological context in which the use of cytokines in combination has evolved to direct a healthy immune tone.

From a translational perspective, cytokine therapies have been limited (56) in part due to the short half-life in circulation $(57,58)$. As such, our findings suggest that IL-2 and IL-4-induced combinatorial signaling may be best harnessed for autologous Treg transfer therapies, which itself has been limited by 
poor expansion and survival of Tregs ex vivo (59). Here, Tregs that underwent stimulation by both IL-2 and IL-4 together not only retained their activated and IL-10-producing state, but also exponentially expanded and survived for almost two weeks in culture with 3 days of cytokine stimulation and 9 days of co-culture with Tconv cells (Figure 3J). Our data further suggests that the IL-2 and IL-4 combination reprograms Tregs with a particular receptor expression and STAT signaling pattern, potentially allowing for the cells to continue suppressing for extended periods through enhanced sensitivity to both cytokines.

In conclusion, we identified a novel mechanism of cell signaling integration controlling the transcriptional and proliferative response of regulatory $T$ cells. While this is demonstrated in $T$ cells using only two cytokines, these findings suggest that combinatorial signaling with other cytokines and cell types is likely. Moreover, while these differences in response may underlie cytokine pleiotropy, they also provide an intriguing guide for new therapeutic applications of combinatorial cytokines in the clinical setting ranging from ex vivo support of autologous cell transfers to immune activation in cancer and 374 inhibition for autoimmunity. 


\section{Materials and Methods}

Mice. $\mathrm{C} 57 \mathrm{BL} / 6 \mathrm{~J}$ (Stock \#000664), IL-10 ${ }^{\mathrm{eGFP}}\left(\mathrm{B} 6.129 \mathrm{~S} 6-\mathrm{II} 10^{\text {tm1Flv }} / \mathrm{J}\right.$, Stock \#008379), FoxP3 ${ }^{\mathrm{RFP}}$ (C57BL/6FoxP3 ${ }^{\text {tm1Flv }} / \mathrm{J}$, Stock \#008374), and IL-10-null (B6.129P2-II10 ${ }^{\text {tm1cgn }} / \mathrm{J}$, Stock \#002251) mice, all on the C57BL/6 background, as well as FoxP3 ${ }^{\text {eGFP }}$ mice on the BALB/c background (Foxp3 ${ }^{\text {tm2Tch }}$ Stock \#006769) were purchased from the Jackson Laboratory (Bar Harbor, ME). IL-10 ${ }^{\text {eGFP }}$ and FoxP3 ${ }^{\text {RFP }}$ mice were crossed to make IL-10 ${ }^{\mathrm{eGFP}} \times$ FoxP3 $^{\mathrm{RFP}}$ dual-reporters in our facility. Mice were housed in a $12 \mathrm{hr}$ light/dark cycle specific pathogen free facility and fed standard chow (Purina 5010) ad libitum. Enrichment and privacy were provided in mating cages by 'breeding huts' (Bio-Serv S3352-400). Mouse studies and all animal housing at Case Western Reserve University were approved by and performed according to the guidelines established by the Institutional Animal Care and Use Committee of CWRU.

Primary splenic $\boldsymbol{T}$ cells. Primary splenocytes were isolated from freshly harvested mouse spleens, and reduced to a single cell suspension by passing them through a sterile $100 \mu \mathrm{M}$ nylon mesh cell strainer (Fisher Scientific, Hampton, NH). The single cell suspensions were labeled with anti-mouse CD4 magnetic microbeads (Miltenyi Biotec, San Diego, CA), and separated with an AutoMACS Pro Separator (Miltenyi Biotec) per manufacturer's instructions.

Cell culture. After flow sorting, Tregs or Tconv cells were cultured in 96-well round bottom plates (Corning, Corning, NY) at 50,000 cells per well in advanced RPMI (Gibco/Fisher Scientific, Waltham, MA) supplemented with $5 \%$ Australian-produced heat-inactivated fetal bovine serum, $55 \mu \mathrm{M} \beta$ mercaptoethanol, $100 \mathrm{U} / \mathrm{mL}$ and $100 \mu \mathrm{g} / \mathrm{mL}$ Penicillin/Streptomycin, and $0.2 \mathrm{mM}$ L-glutamine (Gibco/Fisher Scientific, Waltham, MA) at $5 \% \mathrm{CO} 2,37^{\circ} \mathrm{C}$. For activating conditions, wells were coated with $\alpha C D 3 \varepsilon$ (eBioscience, San Diego, CA) at $2.5 \mu \mathrm{g} / \mathrm{mL}$ in PBS then incubated at $4^{\circ} \mathrm{C}$ overnight followed by two washes with PBS before receiving cells. As indicated, cultures were supplemented with recombinant IL-2 (R\&D Systems, Minneapolis, MN), IL-4 (R\&D Systems), and IL-13 (Invitrogen, Carlsbad, CA), with equimolar concentration being 728 pM. For experiments involving STAT5 inhibitor, STAT5i (Stemcell, Vancouver, BC, CAN) resuspended in DMSO was added directly to culture at the designated amounts. Due to the known toxicity of DMSO, controls with DMSO only were also included, with \% inhibition calculated compared to the DMSO controls.

In vitro Tconv suppression. Tregs were pre-stimulated with $\alpha \mathrm{CD} 3 \varepsilon$ and all combinations of cytokine for 3 or 7 days, washed, then mono-cultured or introduced into co-culture with 50,000 freshly isolated and CellTrace-stained Tconv cells at a 1:2, 1:4, 1:8, 1:16, 1:32, and 1:64 dilution (60).. To assess the suppressive ability of a Treg on a per cell basis, Tregs were counted after stimulation and before placing in co-culture. To determine the suppressive ability of Tregs on a population level, Tregs were counted prior to stimulation so that the number of cells introduced into co-culture with Tconv reflected the proliferation induced by the cytokine conditions. CellTrace signal in Tconv cells was detected by flow cytometry after 3 days of co-culture, and secretion of cytokines were quantified by ELISA.

EAE model of multiple sclerosis. Age-matched female mice between 11-23 weeks underwent EAE induction using the $\mathrm{MOG}_{35-55}$ /CFA Emulsion and pertussis toxin (PTX) kit from Hooke Laboratories (Lawrence, MA) according to manufacturer's instructions. Mice were immunized with $200 \mu \mathrm{g}$ of $\mathrm{MOG}_{35-}$ ${ }_{55} /$ CFA emulsion s.c. in 2 locations in the back on day 0 and were administered $100 \mathrm{ng}$ of PTX i.p. on days 0 and 1 of the trial. Negative controls for disease received a CFA emulsion and PTX according to the same schedule. Mice were weighed and scored daily according to Hooke Laboratory's EAE scoring guide (https://hookelabs.com/protocols/eaeAl_C57BL6.html). Treated mice received cytokine cocktail 
comprising of equimolar IL-2 (20 ng) and IL-4 (16.24 ng) i.v. in a volume of $100 \mu$ l of sterile PBS every other day. Untreated control mice received $100 \mu \mathrm{l}$ of sterile PBS i.v. at the same time intervals.

HDM and TAC models of pulmonary inflammation. Age and sex-matched mice between 8-23 weeks were challenged with house dust mite antigen (HDM, D. Farinae, GREER, Lenoir, NC) by i.n. delivery of $20 \mu \mathrm{g} \mathrm{HDM} /$ dose in PBS according to the designated challenge schedule (Figures 5A, 5C, 6A) and sacrificed accordingly (61). IL-2 and IL-4 at the designated doses and time intervals were delivered i.n. in a mixed cocktail according after animals were anesthetized with $3 \%$ isoflurane (Baxter, Deerfield, IL) with an anesthesia system (VetEquip, Livermore, CA). In the chronic triple antigen combination model, mice were sensitized intraperitoneally with $20 \mu \mathrm{g}$ Ovalbumin (OVA, Albumin from chicken egg white, Millipore Sigma, Darmstadt, Germany), $2.5 \mu \mathrm{g}$ cockroach antigen (CRA, American, Periplaneta Americana, GREER, Lenoir, NC) and $2.5 \mu \mathrm{g}$ of HDM in PBS. Immediately following sensitization, mice were challenged intranasally with HDM, CRA, or OVA in a rotating schedule for 7 weeks (62). For Flexivent (SCIREQ, Montreal, Quebec) studies, mice were anesthetized with a cocktail of Ketamine/Xylazine/Acepromazine, the trachea was exposed and catheterized, methacholine was administered via nebulization in increasing doses. Airway resistance was measured on a Flexivent respirator over time. After mice were euthanized, bronchial alveolar lavage fluid (BALf) was recovered in 3 lavages of $1 \mathrm{ml}$ each, and lung tissues underwent FFPE processing or were reduced to a single cell suspension for flow cytometric analysis. BALf cell differentials were acquired by a HemaVet 950 Hematology Analyzer.

Flow cytometry and cell sorting. For splenic Treg cell sorting, magnetic bead-mediated positively selected $\mathrm{CD}^{+}$cells were sorted by endogenously expressed FoxP3 ${ }^{\mathrm{RFP}}$ and/or IL-10 ${ }^{\mathrm{GFP}}$. For IL-10ko mice, $\mathrm{CD}^{+}$cells were stained and sorted with CD25-BV421 (BD Biosciences, San Jose, CA). Cells were washed in MACS buffer (Miltenyi Biotec) before sterile cell sorting using a FACSAria-SORP (BD Biosciences). For flow cytometric analysis of tissue compartments, cells were passed through a sterile $70 \mu \mathrm{M}$ nylon mesh cell strainer (Fisher Scientific, Hampton, $\mathrm{NH}$ ) for homogenization into a single cell suspension. Blood and spleen were RBC-lysed (BD, Franklin Lakes, NJ), and brain and spinal underwent a 30\%/70\% Percoll gradient centrifugation (GE, Boston, MA). For intracellular staining, cells were fixed and permeabilized with the FoxP3/transcription factor staining buffer set (eBioscience). Commercial antibodies used included: CD4-PE (eBioscience), CD4 - AF647 (Biolegend), F4/80 - BV711 (BD), CD25-BV421 (BD), CD25PE (eBioscience), CD25 - PE-Cy7 (BD), CD124 - BB700 (BD), CD122 - APC (BD), CD132 - BV421 (BD), CD213a1 - Biotin (MyBioSource, San Diego, CA), Biotin - BV-605 (BD), STAT3 - AF647 (BD), STAT5 AF647 (BD), STAT6 - AF647 (BD) and mTOR - AF647 (BD). Additional flow cytometry reagents purchased commercially include: Sytox Red (Invitrogen), CellTrace Violet (Invitrogen), and Fixable Viability Stain 510 (BD). Tetramers obtained from the NIH Tetramer Core include MOG $_{35-55}$ Tetramer - BV421, I-A(b) Tetramer - BV421 (Atlanta, GA). Cells were acquired on an Attune NxT cytometer (Invitrogen). Cytometer usage was supported by the Cytometry \& Imaging Microscopy Core Facility of the Case Comprehensive Cancer Center. Analysis of all FACS data was performed using FlowJo v10 (Tree Star, Inc., Ashland, OR). Analysis of proliferation was done in FlowJo v10 using the Proliferation Modeling platform.

Cell Proliferation. Cell proliferation was quantified using CellTrace Violet staining coupled with analysis of dilution using flow cytometry. Division Indices were calculated mathematically using the Proliferation Modeling platform in Flowjo by dividing the total number of cell divisions by the total number of cells in the starting culture: , where =generation. Proliferation Indices were calculated by dividing the total number of divisions by the number of cells that underwent division: . 
ELISA and Luminex. Cytokine concentrations were analyzed by sandwich ELISA performed according to manufacturer's instructions (BioLegend). The assay was modified to utilize europium-conjugated streptavidin (Perkin-Elmer, San Jose, CA). Signal was detected with a Victor V3 plate reader (Perkin Elmer). For Luminex assays, media were snap frozen in liquid nitrogen and sent to Eve Technologies (Calgary, AB) for mouse 31-plex and TGF $\beta$ 3-plex analysis.

467

468

469

470

471

472

473

474

475

476

477

478

479

480

481

482

483

484

485

486

Histology and microscopy. Harvested tissues were fixed in 10\% formalin (VWR, Radnor, PA) for 24 hours and sent to AML Laboratories (Jacksonville, FL) for paraffin embedding. Embedded lung tissues were sectioned and stained with H\&E, Periodic Acid Schiff (PAS), or trichrome by AML and spinal cord tissues were sectioned and stained with Hematoxyling and Eosin (H\&E) (Ricca Chemical, Arlington, TX and Sigma Aldrich, St. Louis, MO) or Luxol Fast Blue (LFB) (Sigma Aldrich) in our lab. Images were acquired with a Leica DM IL LED (Wetzlar, Germany).

Power Analyses. Hooke Laboratories established that 10-12 mice are needed in MOG challenge groups using their strain-specific standardized reagents and procedures to account for the natural scatter of this pre-clinical disease model. For the asthma models, we used historical means between positive and negative control groups and their associated standard deviation to establish statistical power in animal model experimental design. The power was set to 0.80 with $\alpha=0.05$ in a 2 -sided test at https://www.stat.ubc.ca/ rollin/stats/ssize/n2.html). We found that 6 mice were sufficient per group.

Data Analyses. All data are represented by mean \pm SEM. Pulmonary inflammation data sets include a minimum of four, but more commonly six animals per group per experiment. EAE trials include four animals in the negative disease control conditions and 10-12 animals in groups that receive MOG peptide. Data and statistical measurements were generated with Prism (Graphpad, San Digo, CA). For comparisons between two groups, Student's t-test was used; comparisons between multiple groups utilized analysis of variance. The Principle Component analysis was generated in RStudio using factoextra and ggplot2. Mean \pm SEM are indicated. ${ }^{*} p<0.05,{ }^{* *} p<0.01,{ }^{* * *} p<0.001,{ }^{* * * *} p<0.0001$. 


\section{Supplementary Materials}

490 Figure 1 - figure supplement 1. Cell sorting gating strategy and post-sort purity

491 Figure 1 - figure supplement 2. IL-10 is the only synergistic and robust analyte produced by Tregs

492 following combinatorial cytokine stimulation

493 Figure 1 - figure supplement 3. Dynamics of combinatorial cytokine stimulation of Tregs over time

494 Figure 4 - figure supplement 1. The IL-10 and proliferative response following combined IL-2 and IL-4

495 stimulation is dependent on STAT5 signaling

496 Figure 6 - figure supplement 1. Administration of IL-2 and IL-4 suppresses HDM-induced acute asthma

497 and is dependent on IL-10

498 Figure 6 - figure supplement 2. Administration of IL-2 and IL-4 therapeutically suppresses TAC-induced 499 chronic asthma

500 Figure 7 - figure supplement 1. Combined IL-2 and IL-4 suppressed the severity of EAE in Preventative, 501 Concomitant, and Therapeutic dosing regimen 


\section{$\underline{\text { References }}$}

$503 \quad$ 1. W. J. Leonard, J. X. Lin, J. J. O'Shea, The gammac Family of Cytokines: Basic Biology to Therapeutic Ramifications. Immunity 50, 832-850 (2019).

2. D. A. Morgan, F. W. Ruscetti, R. Gallo, Selective in vitro growth of T lymphocytes from normal human bone marrows. Science 193, 1007-1008 (1976). W. Liao, J. X. Lin, W. J. Leonard, Interleukin-2 at the
and immunotherapy. Immunity 38, 13-25 (2013).

3. W. Liao, J. X. Lin, W. J. Leonard, Interleukin-2 at the crossroads of effector responses, tolerance,

4. C. S. Henney, K. Kuribayashi, D. E. Kern, S. Gillis, Interleukin-2 augments natural killer cell activity. Nature 291, 335-338 (1981).

5. J. P. Siegel, M. Sharon, P. L. Smith, W. J. Leonard, The IL-2 receptor beta chain (p70): role in mediating signals for LAK, NK, and proliferative activities. Science 238, 75-78 (1987).

6. M. C. Mingari et al., Human interleukin-2 promotes proliferation of activated B cells via surface receptors similar to those of activated T cells. Nature 312, 641-643 (1984).

7. Y. Refaeli, L. Van Parijs, C. A. London, J. Tschopp, A. K. Abbas, Biochemical Mechanisms of IL-2Regulated Fas-Mediated T Cell Apoptosis. Immunity 8, 615-623 (1998).

8. T. Chinen et al., An essential role for the IL-2 receptor in Treg cell function. Nat Immunol 17, 1322-1333 (2016).

9. I. Horak, Immunodeficiency in IL-2-knockout mice. Clinical Immunology and Immunopathology 76, S172-S173 (1995).

10. T. R. Malek, The biology of interleukin-2. Annu Rev Immunol 26, 453-479 (2008).

11. R. Spolski, D. Gromer, W. J. Leonard, The gamma c family of cytokines: fine-tuning signals from IL-2 and IL-21 in the regulation of the immune response. F1000Res 6, 1872 (2017).

12. T. A. Waldmann, The multi-subunit interleukin-2 receptor. Annu Rev Biochem 58, 875-911 (1989).

13. J. A. Johnston et al., Tyrosine phosphorylation and activation of STAT5, STAT3, and Janus kinases by interleukins 2 and 15. Proc Natl Acad Sci U S A 92, 8705-8709 (1995).

14. K. Nelms, A. D. Keegan, J. Zamorano, J. J. Ryan, W. E. Paul, The IL-4 receptor: signaling mechanisms and biologic functions. Annu Rev Immunol 17, 701-738 (1999).

15. J. Hou et al., An interleukin-4-induced transcription factor: IL-4 Stat. Science 265, 1701-1706 (1994).

16. S. P. Gadani, J. C. Cronk, G. T. Norris, J. Kipnis, IL-4 in the brain: a cytokine to remember. J Immunol 189, 4213-4219 (2012).

17. R. S. Geha, H. H. Jabara, S. R. Brodeur, The regulation of immunoglobulin E class-switch recombination. Nat Rev Immunol 3, 721-732 (2003).

18. J. F. Urban, Jr., I. M. Katona, W. E. Paul, F. D. Finkelman, Interleukin 4 is important in protective immunity to a gastrointestinal nematode infection in mice. Proc Natl Acad Sci U S A 88, 55135517 (1991).

19. W. E. Paul, History of interleukin-4. Cytokine 75, 3-7 (2015).

20. S. Gordon, Alternative activation of macrophages. Nat Rev Immunol 3, 23-35 (2003).

21. A. Vogelgesang, S. Rosenberg, S. Skrzipek, B. M. Broker, A. Dressel, Mitoxantrone treatment in multiple sclerosis induces TH2-type cytokines. Acta Neurol Scand 122, 237-243 (2010).

22. C. Weigert, M. Rocken, K. Ghoreschi, Interleukin 4 as a potential drug candidate for psoriasis. Expert Opin Drug Discov 3, 357-368 (2008).

23. P. Krzyszczyk, R. Schloss, A. Palmer, F. Berthiaume, The Role of Macrophages in Acute and Chronic Wound Healing and Interventions to Promote Pro-wound Healing Phenotypes. Front Physiol 9, 419 (2018). 
24. R. E. Mitchell et al., IL-4 enhances IL-10 production in Th1 cells: implications for Th1 and Th2 regulation. Sci Rep 7, 11315 (2017).

25. J. S. Rawlings, K. M. Rosler, D. A. Harrison, The JAK/STAT signaling pathway. J Cell Sci 117, 12811283 (2004).

26. J. J. O'Shea et al., The JAK-STAT pathway: impact on human disease and therapeutic intervention. Annu Rev Med 66, 311-328 (2015).

27. J. X. Lin, W. J. Leonard, Fine-Tuning Cytokine Signals. Annu Rev Immunol 37, 295-324 (2019).

28. J. Zhu, L. Guo, C. J. Watson, J. Hu-Li, W. E. Paul, Stat6 is necessary and sufficient for IL-4's role in Th2 differentiation and cell expansion. J Immunol 166, 7276-7281 (2001).

29. M. B. Jones et al., CD45Rb-low effector T cells require IL-4 to induce IL-10 in FoxP3 Tregs and to protect mice from inflammation. PLoS One 14, e0216893 (2019).

30. Y. Y. Wan, R. A. Flavell, Identifying Foxp3-expressing suppressor T cells with a bicistronic reporter. Proc Natl Acad Sci U S A 102, 5126-5131 (2005).

31. M. Kamanaka et al., Expression of interleukin-10 in intestinal lymphocytes detected by an interleukin-10 reporter knockin tiger mouse. Immunity 25, 941-952 (2006).

32. D. Q. Tran, TGF-beta: the sword, the wand, and the shield of FOXP3(+) regulatory T cells. J Mol Cell Biol 4, 29-37 (2012).

33. C. A. Akdis, K. Blaser, Mechanisms of interleukin-10-mediated immune suppression. Immunology 103, 131-136 (2001).

34. K. Takeda et al., Essential role of Stat6 in IL-4 signalling. Nature 380, 627-630 (1996).

35. K. Tsuji-Takayama et al., The production of IL-10 by human regulatory T cells is enhanced by IL-2 through a STAT5-responsive intronic enhancer in the IL-10 locus. Journal of Immunology 181, 3897-3905 (2008).

36. H.-P. Kim, J. Kelly, W. J. Leonard, The Basis for IL-2-Induced IL-2 Receptor $\alpha$ Chain Gene Regulation. Immunity 15, 159-172 (2001).

37. J. L. Johnson, M. B. Jones, B. A. Cobb, Polysaccharide-experienced effector T cells induce IL-10 in FoxP3+ regulatory T cells to prevent pulmonary inflammation. Glycobiology 28, 50-58 (2018).

38. J. L. Johnson, M. B. Jones, B. A. Cobb, Bacterial capsular polysaccharide prevents the onset of asthma through T-cell activation. Glycobiology 25, 368-375 (2015).

39. J. L. Johnson, M. B. Jones, B. A. Cobb, Polysaccharide A from the capsule of Bacteroides fragilis induces clonal CD4+ T cell expansion. J Biol Chem 290, 5007-5014 (2015).

40. P. Loke et al., IL-4 dependent alternatively-activated macrophages have a distinctive in vivo gene expression phenotype. BMC Immunol 3, 7 (2002).

41. R. J. Johnston, Y. S. Choi, J. A. Diamond, J. A. Yang, S. Crotty, STAT5 is a potent negative regulator of TFH cell differentiation. J Exp Med 209, 243-250 (2012).

42. W. Liao, J. X. Lin, W. J. Leonard, IL-2 family cytokines: new insights into the complex roles of IL-2 as a broad regulator of T helper cell differentiation. Curr Opin Immunol 23, 598-604 (2011).

43. J. Cote-Sierra et al., Interleukin 2 plays a central role in Th2 differentiation. Proc Natl Acad Sci U SA 101, 3880-3885 (2004).

44. J. Zhu, J. Cote-Sierra, L. Guo, W. E. Paul, Stat5 activation plays a critical role in Th2 differentiation. Immunity 19, 739-748 (2003).

45. Y. Komiyama et al., IL-17 Plays an Important Role in the Development of Experimental Autoimmune Encephalomyelitis. The Journal of Immunology 177, 566-573 (2006).

46. M. M. Gueders et al., Mouse models of asthma: a comparison between C57BL/6 and BALB/C strains regarding bronchial responsiveness, inflammation, and cytokine production. Inflamm Res 58, 845-854 (2009). 
600

601

602

603

604

605

606

607

608

609

610

611

612

613

614

615

616

617

618

619

620

621

622

623

624

625

626

627

628

629

630

631

632

633

634

635

636

47. J. W. Steinke, L. Borish, Th2 cytokines and asthma. Interleukin-4: its role in the pathogenesis of asthma, and targeting it for asthma treatment with interleukin-4 receptor antagonists. Respir Res 2, 66-70 (2001).

48. A. G. Wexler, A. L. Goodman, An insider's perspective: Bacteroides as a window into the microbiome. Nat Microbiol 2, 17026 (2017).

49. E. B. Troy, D. L. Kasper, Beneficial effects of Bacteroides fragilis polysaccharides on the immune system. Front Biosci (Landmark Ed) 15, 25-34 (2010).

50. J. Ochoa-Reparaz et al., Central nervous system demyelinating disease protection by the human commensal Bacteroides fragilis depends on polysaccharide A expression. J Immunol 185, 41014108 (2010).

51. A. O. Tzianabos, A. B. Onderdonk, D. F. Zaleznik, R. S. Smith, D. L. Kasper, Structural characteristics of polysaccharides that induce protection against intra-abdominal abscess formation. Infect Immun 62, 4881-4886 (1994).

52. G. Sharon et al., Human Gut Microbiota from Autism Spectrum Disorder Promote Behavioral Symptoms in Mice. Cell 177, 1600-1618 e1617 (2019).

53. E. Y. Hsiao et al., Microbiota modulate behavioral and physiological abnormalities associated with neurodevelopmental disorders. Cell 155, 1451-1463 (2013).

54. B. A. Cobb, Q. Wang, A. O. Tzianabos, D. L. Kasper, Polysaccharide processing and presentation by the MHCII pathway. Cell 117, 677-687 (2004).

55. A. O. Tzianabos et al., IL-2 mediates protection against abscess formation in an experimental model of sepsis. J Immunol 163, 893-897 (1999).

56. J. Tomala, H. Chmelova, T. Mrkvan, B. Rihova, M. Kovar, In vivo expansion of activated naive CD8+ T cells and NK cells driven by complexes of IL-2 and anti-IL-2 monoclonal antibody as novel approach of cancer immunotherapy. J Immunol 183, 4904-4912 (2009).

57. J. H. Donohue, S. A. Rosenberg, The fate of interleukin-2 after in vivo administration. $J$ Immunol 130, 2203-2208 (1983).

58. P. J. Conlon, S. Tyler, K. H. Grabstein, P. Morrissey, Interleukin-4 (B-cell stimulatory factor-1) augments the in vivo generation of cytotoxic cells in immunosuppressed animals. Biotechnol Ther 1, 31-41 (1989).

59. Q. Tang, J. A. Bluestone, Regulatory T-cell therapy in transplantation: moving to the clinic. Cold Spring Harb Perspect Med 3, (2013).

60. L. W. Collison, D. A. Vignali, In vitro Treg suppression assays. Methods Mol Biol 707, 21-37 (2011).

61. J. R. Johnson et al., Continuous exposure to house dust mite elicits chronic airway inflammation and structural remodeling. Am J Respir Crit Care Med 169, 378-385 (2004).

62. M. J. Duechs, C. Tilp, C. Tomsic, F. Gantner, K. J. Erb, Development of a novel severe triple allergen asthma model in mice which is resistant to dexamethasone and partially resistant to TLR7 and TLR9 agonist treatment. PLoS One 9, e91223 (2014). 


\section{Acknowledgments}

638 We are immensely grateful for the help of Douglas M. Oswald, PhD, for valuable scientific input and 639 guidance in R, Mark B. Jones, PhD for mentorship and project discussions early on, Kalob M. Reynero for 640 technical assistance in tissue sectioning, Jill M. Cavanaugh for technical assistance and maintenance of 641 the mouse colony, and Lori. S.C. Kreisman for laboratory support and manuscript editing. Moreover, we 642 thank Sandra Siedlak and Xiongwei Zhu, PhD for expertise in histological sectioning and staining, and 643 Alex Huang, MD, PhD for equipment for histological imaging. We thank the Cytometry \& Microscopy 644 Shared Resource of the Case Comprehensive Cancer Center (P30CA043703) for equipment and 645 assistance with flow cytometry-based experiments. This work was made possible by grants from: The 646 National Institutes of Health (R01-GM115234), and the Hartwell Foundation to BAC, and the National 647 Institutes of Health (T32-AI089474) to JYZ and CAA.

\section{Author contributions}

649 Conceptualization, J.Y.Z. and B.A.C.; Methodology, J.Y.Z. and B.A.C.; Investigation, J.Y.Z. and C.A.A.; 650 Formal Analysis, J.Y.Z. and B.A.C.; Writing - Original Draft, J.Y.Z.; Writing - Review \& Editing, J.Y.Z. and 651 B.A.C.; Funding Acquisition, B.A.C.; Resources, B.A.C.; Supervision, B.A.C.

\section{Declaration of Interests}

653 We have no conflicts of interests to declare. 


\section{Abbreviations}

656 IL-2: interleukin-2

657 IL-4: interleukin-4

658 IL-10: Interleukin-10

659 IL-17: interleukin-17

660 IL-2RyC: IL-2 receptor common gamma chain, CD132

661 IL-2R $\alpha$ : IL-2 receptor alpha chain, CD25

662 IL-2Rß: IL-2 receptor beta chain, CD122

663 IL-4R $\alpha$ : IL-4 receptor alpha chain, CD124

664 IL-13R $\alpha 1$ : IL-13 receptor subunit alpha 1, CD213 $\alpha 1$

665 Treg: regulatory $T$ cell

666 Tconv: conventional T cell

667 EAE: experimental autoimmune encephalomyelitis

668 FoxP3: forkhead box P3

669 RFP: red fluorescent protein

670 GFP: green fluorescent protein

671 TCR: T cell receptor

672 PCA: principle component analysis

673 HDM: house dust mite

674 CRA: cockroach antigen

675 OVA: ovalbumin

676 TAC: triple antigen cocktail

677 BALf: bronchial alveolar lavage fluid

678 H\&E: hematoxylin and eosin

679 PAS: periodic acid Schiff

680 LFB: luxol fast blue

681 MOG: myelin oligodendrocyte glycoprotein

682 CFA: complete Freund's adjuvant

683 PTX: pertussis toxin

684 CNS: central nervous system

685 PSA: polysaccharide $A$

686 
bioRxiv preprint doi: https://doi.org/10.1101/2020.04.14.040485; this version posted April 14, 2020. The copyright holder for this preprint (which was not certified by peer review) is the author/funder, who has granted bioRxiv a license to display the preprint in perpetuity. It is made available under aCC-BY 4.0 International license.

\section{$687 \quad$ Figures}

\section{Figure 1}

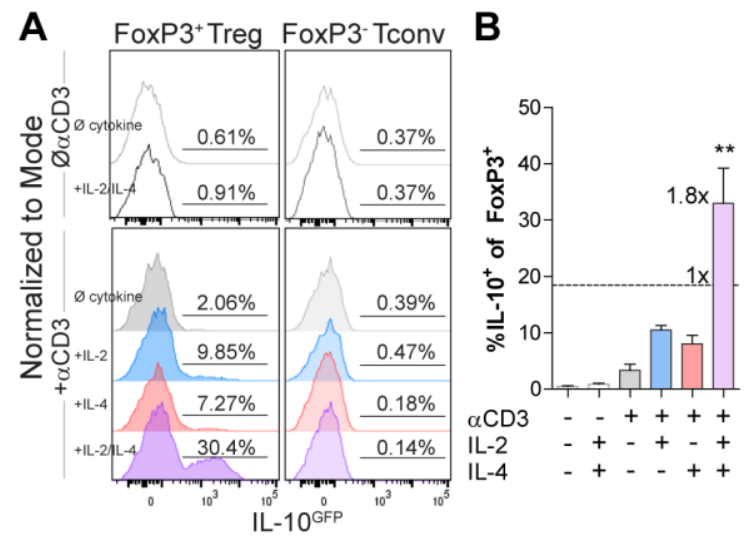

C

D

E

F

G
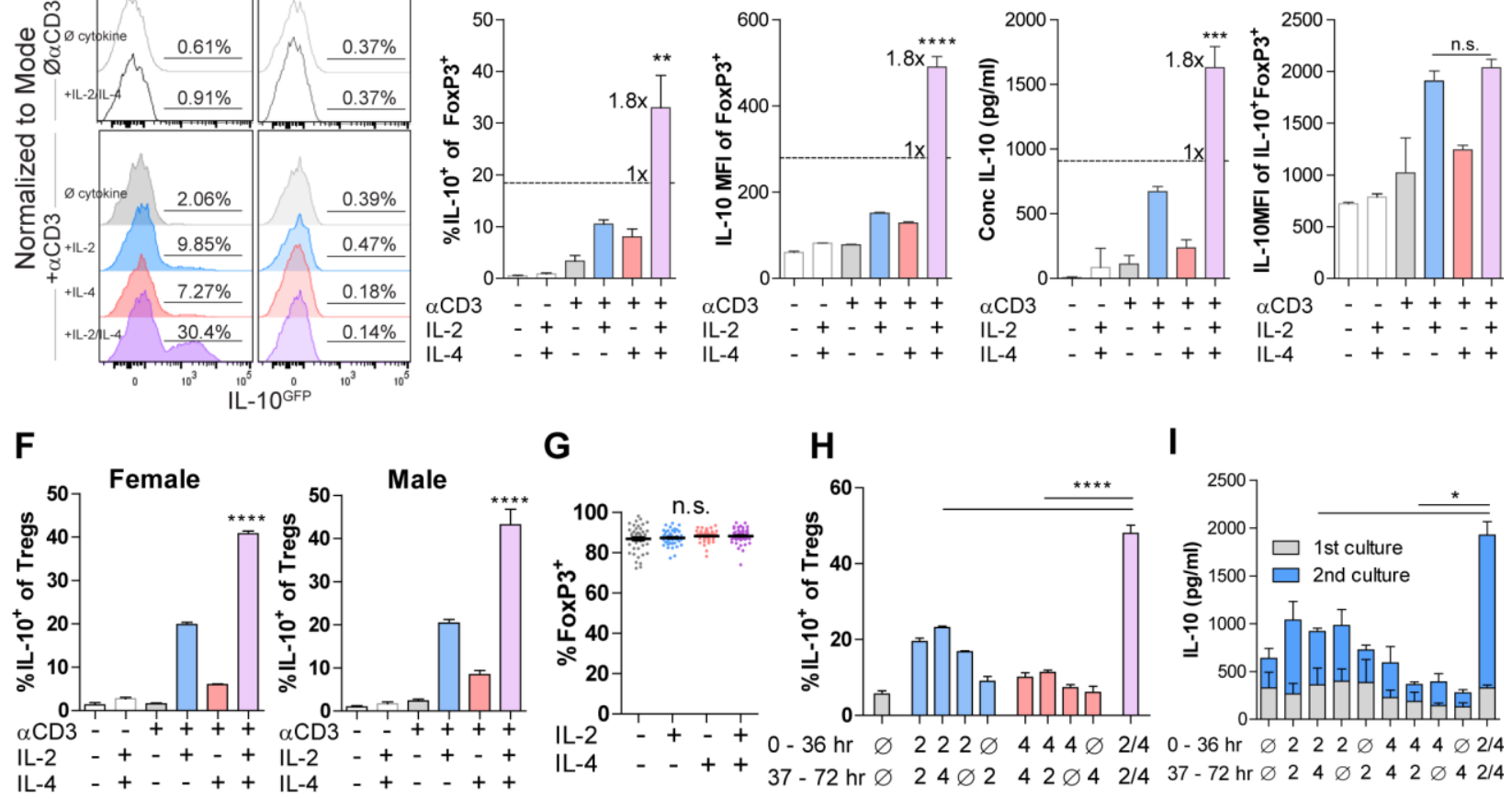

J

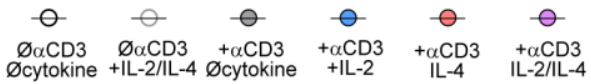

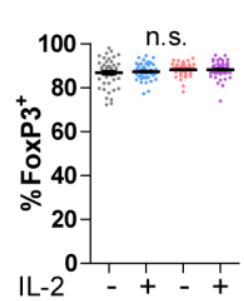

H I
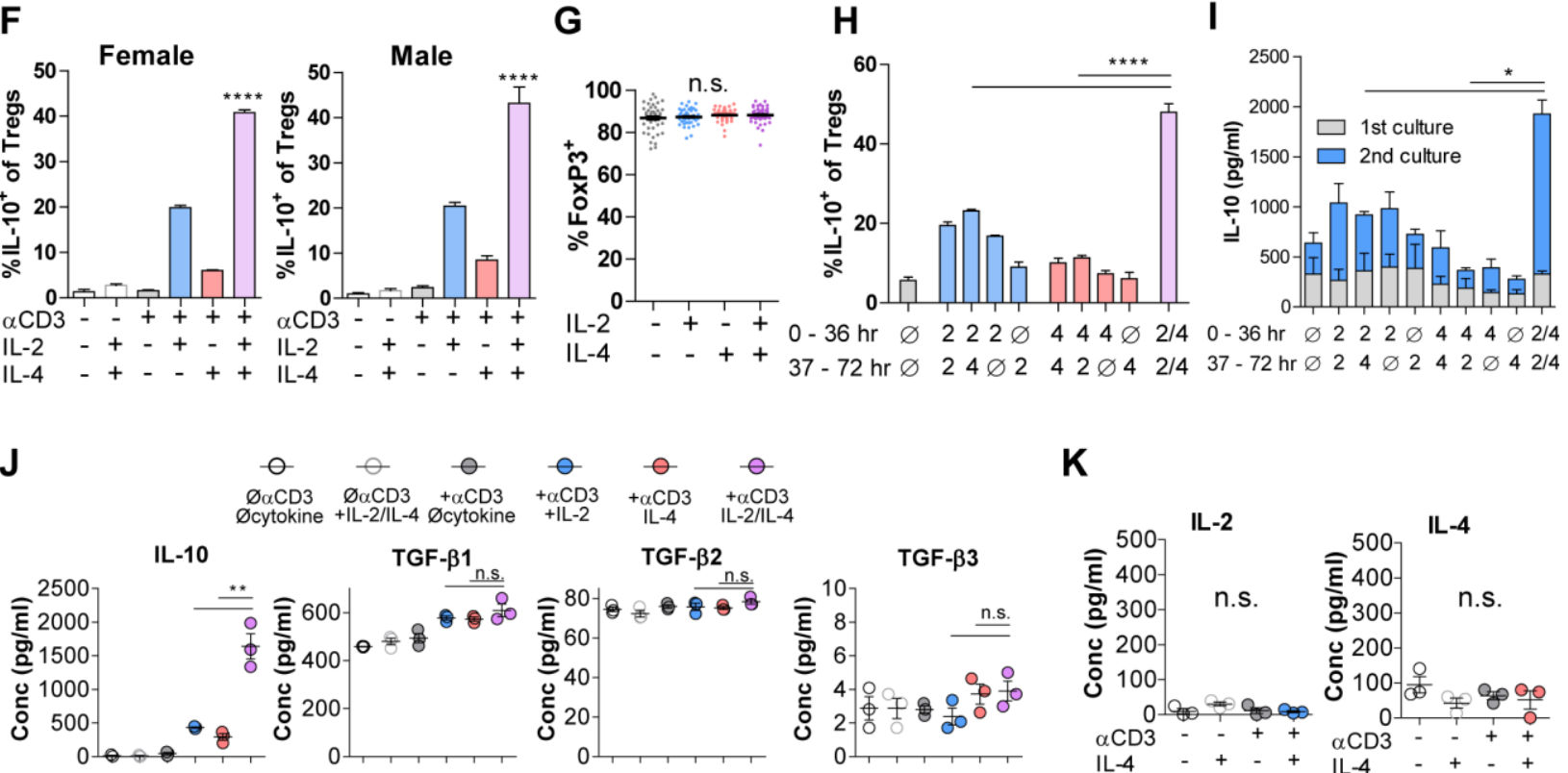

$\mathbf{L}$

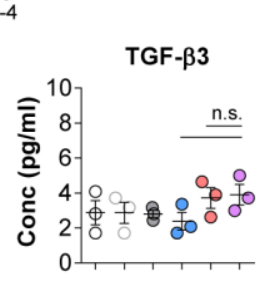

K

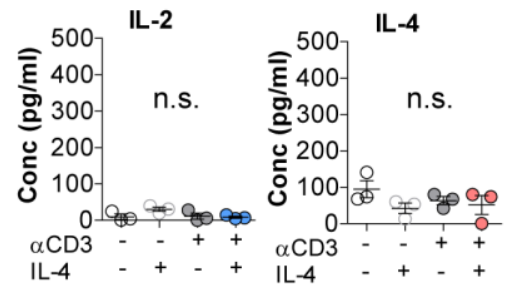

Day 0

Day 3
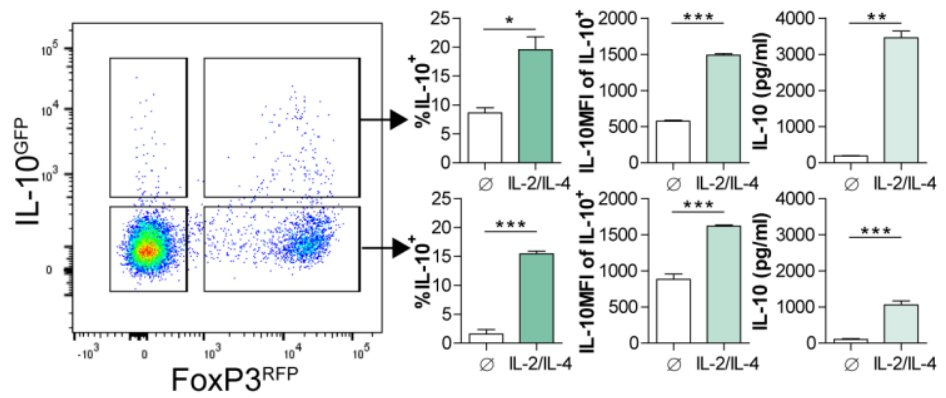

M

Figure 1. IL-2 and IL-4 synergistically promote IL-10 production by Tregs

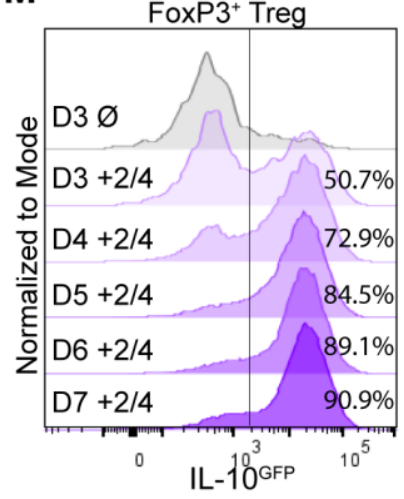


691 (A-C) IL-10 expression of Tregs purified from FoxP3 ${ }^{\text {RFP }} /$ IL-10 ${ }^{\text {GFP }}$ dual reporter mice (See also Figure 1 692 figure supplement 1) cultured for 3 days with the designated stimulants as analyzed by flow cytometry.

693 For all panels, $\mathrm{N} \geq 3$ for all bar graphs and histograms are representative.

694 (D) IL-10 production of Tregs cultured with the designated stimulation as quantified by ELISA of the 695 culture supernatants. $\mathrm{N}=3$.

696 (E) IL-10 expression of purified IL-10 $10^{+}$Tregs cultured with the designated stimulation as quantified by 697 flow cytometry. $\mathrm{N}=3$

698 (F) Female and male responses to combinatorial cytokine stimulation after 3 days, as measured by flow 699 cytometry for IL-10 expression. $\mathrm{N}=3$.

700 (G) FoxP3 expression by purified Tregs stimulated in culture for 3 days with the designated conditions, as 701 analyzed by flow cytometry. $\mathrm{N} \geq 27$.

702 (H) IL-10 expression of purified Tregs stimulated for 36 hours in culture, washed, then subsequently 703 stimulated for another 36 hours in culture with the indicated conditions, as analyzed by flow cytometry. All samples received $\alpha \mathrm{CD} 3 \varepsilon$ activation. (See also Figure 1 - figure supplement $3 A$ ). $N=3$

(I) IL-10 production by purified Tregs stimulated for 36 hours in culture, washed, then subsequently stimulated for another 36 hours in culture with the indicated conditions, as analyzed by ELISA of the culture supernatants. All samples received $\alpha \mathrm{CD} 3 \varepsilon$ activation. (See also Figure 1 - figure supplement 3B).

$708 \mathrm{~N}=3$

709 (J) Cytokine production following 3 days of Treg culture as quantified by multianalyte Luminex of the culture supernatants (See also Figure 1 - figure supplement 2). $\mathrm{N}=3$

711 (K) IL-2 and IL-4 production by Tregs following 3 days of stimulation with the designated conditions, as 712 quantified by ELISA of the culture supernatants. $\mathrm{N}=3$

713 (L) IL-10 production of purified IL-10+ or IL-10- Tregs following 3 days of culture with $\alpha \mathrm{CD} 3 \varepsilon$ and combined IL-2/IL-4, as analyzed by flow cytometry. (See also Figure 1 - figure supplement $3 \mathrm{C}$ ). $\mathrm{N}=3$ (M) IL-10 production of purified Tregs cultured with $\alpha$ CD3 $\varepsilon$ and combined IL-2/IL-4 for 3 to 7 days as analyzed by flow cytometry (See also Figure 1 - figure supplement 3D). Histograms are representative of 7173 independent experiments.

718 Mean \pm SEM are indicated. ${ }^{*} p<0.05,{ }^{* *} p<0.01,{ }^{* * *} p<0.001, * * * * p<0.0001$ 
bioRxiv preprint doi: https://doi.org/10.1101/2020.04.14.040485; this version posted April 14, 2020. The copyright holder for this preprint (which was not certified by peer review) is the author/funder, who has granted bioRxiv a license to display the preprint in perpetuity. It is made available under aCC-BY 4.0 International license.

Figure 2

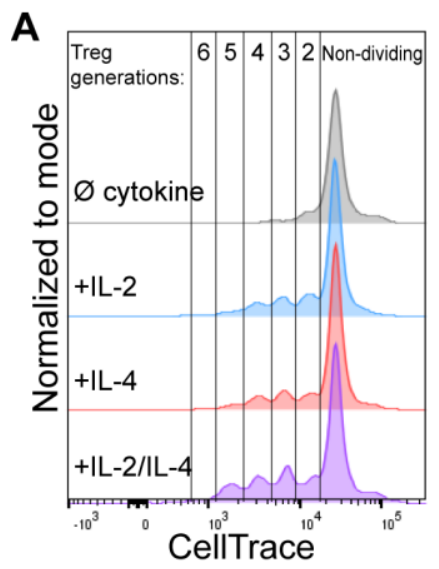

B

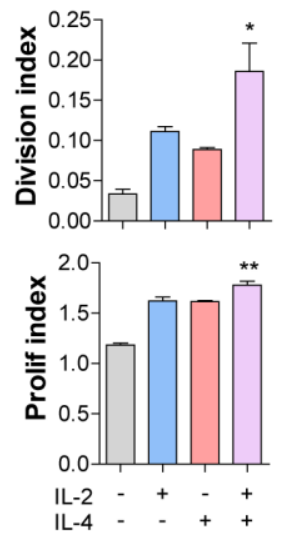

C

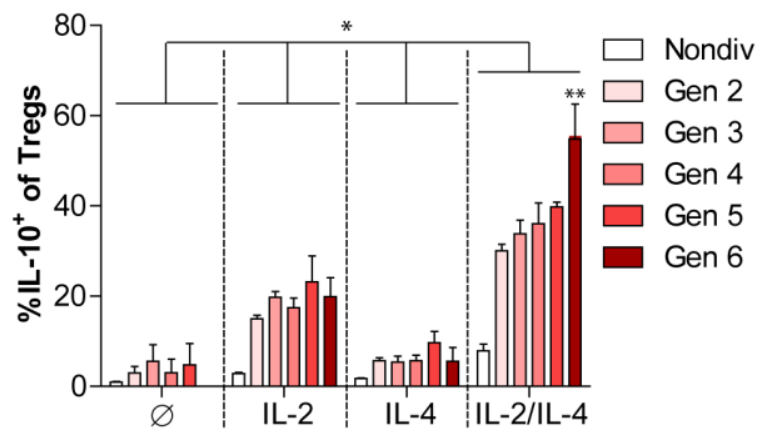

D
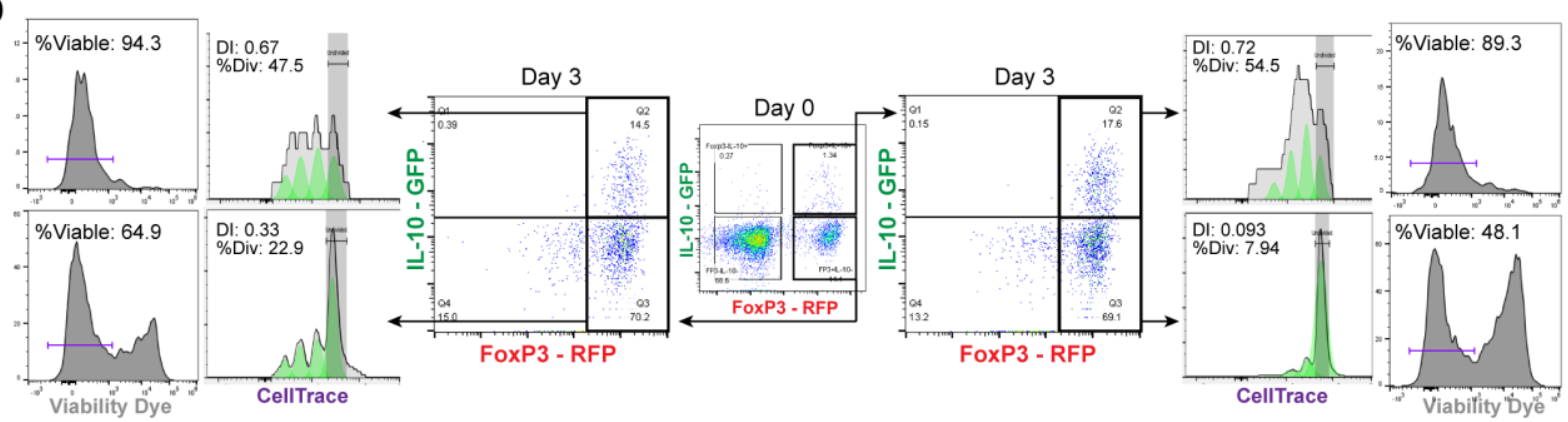

E

$\mathbf{F}$

IL-10-Treg IL-10+Treg $\mathbf{G}$
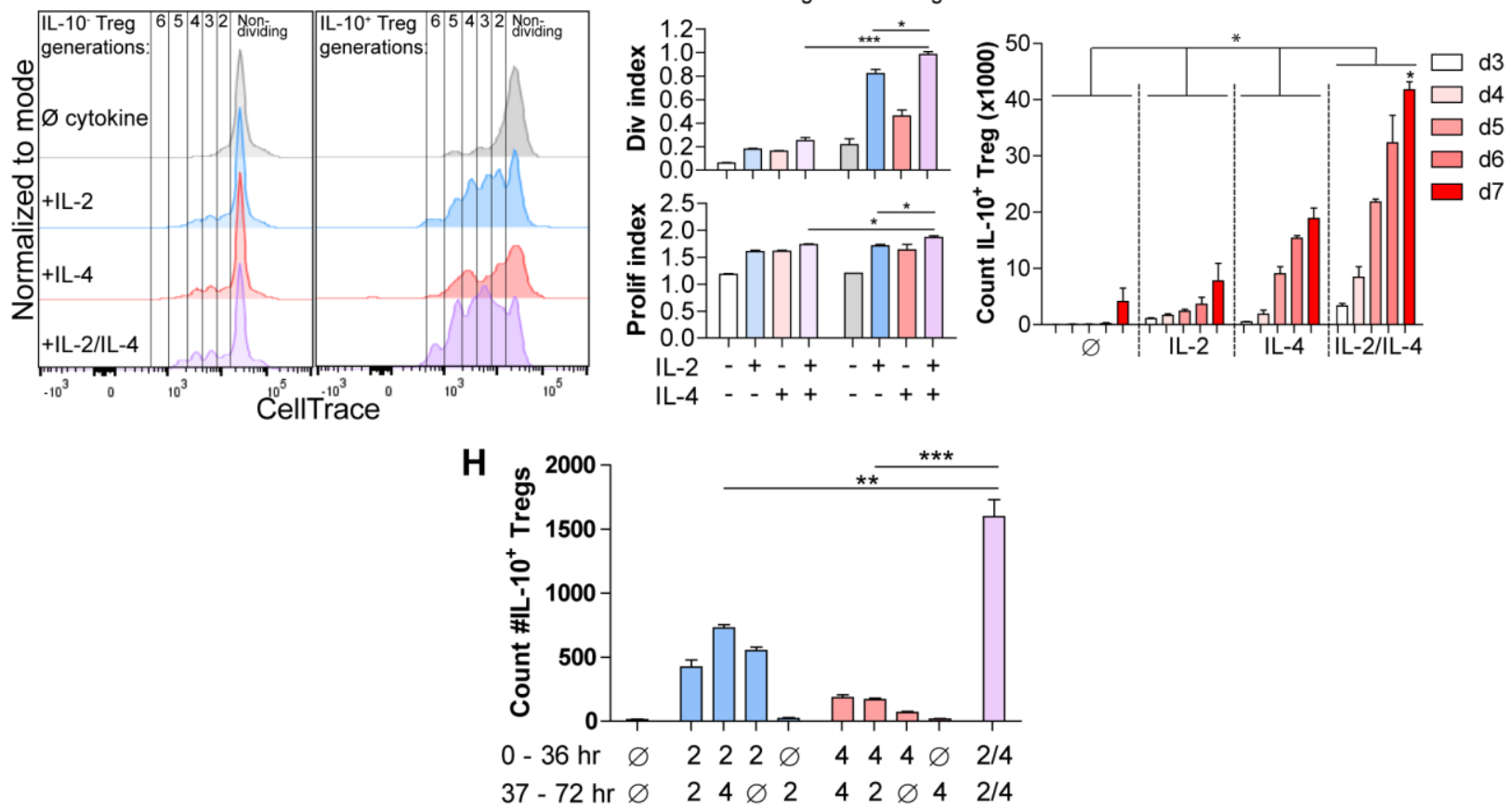

Figure 2. IL-2/IL-4 enhances Treg proliferation and selectively drives the expansion of IL-10 ${ }^{+}$Tregs 
(A-B) Treg proliferation as measured by CellTrace signal following 3 days of culture with $\alpha \mathrm{CD} 3 \varepsilon$ and all combinations of IL-2 and IL-4, with Division and Proliferation Indices indicated. N=3 for all bar graphs and histograms are representative.

(C) IL-10 expression of purified Tregs cultured for 3 days with $\alpha \mathrm{CD} 3 \varepsilon$ and the designated cytokines, and gated by CellTrace generation, as measured by flow cytometry (panel A). $\mathrm{N}=3$. The IL-10 expression of all IL-2/IL-4-induced Treg generations are statistically significant $(p<0.05)$ compared to the other cytokinestimulated conditions.

(D) Proliferation and viability of purified IL-10 ${ }^{+}$and IL-10- Tregs cultured for 3 days with $\alpha \mathrm{CD} 3 \varepsilon$ and both IL-2 and IL-4, as flow cytometry analysis of CellTrace and Sytox Red signal. The histograms are representative. $\mathrm{N}=3$ gated on $\mathrm{IL}-10^{+/}$expression, as analyzed by flow cytometry. $\mathrm{N}=3$ for all bar graphs and histograms are representative.

740 (G) Cell count of IL-10+ Tregs following 3-7 days of culture with $\alpha$ CD3 $\varepsilon$ and all combinations of IL-2 and $741 \mathrm{IL}-4$, as analyzed by flow cytometry. $\mathrm{N}=3$. The cell counts of all IL-2/IL-4-induced conditions are 742 statistically significant $(p<0.05)$ compared to the other cytokine-stimulated conditions.

743 (H) Enumeration of purified Tregs that underwent stimulation with $\alpha C D 3 \varepsilon$ and all cytokine combinations 744 for 36 hours in culture with the first stimulation conditions, and another 36 hours with the second 745 stimulation conditions after washing. Cells underwent flow cytometry. $\mathrm{N}=3$.

746 For all panels, mean \pm SEM are indicated. ${ }^{*} p<0.05,{ }^{* *} p<0.01,{ }^{* * *} p<0.001$ 


\section{Figure 3}

A

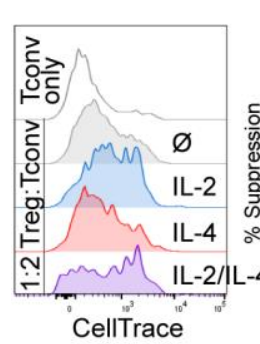

WT Tregs

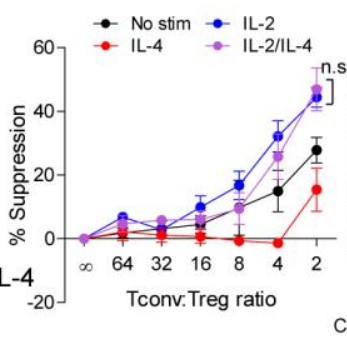

D IL-10-1/ Tregs

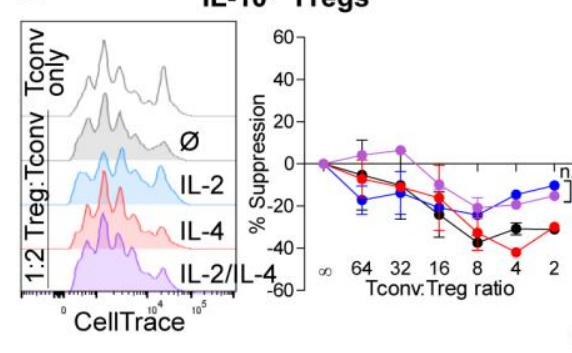

G WT 7d population
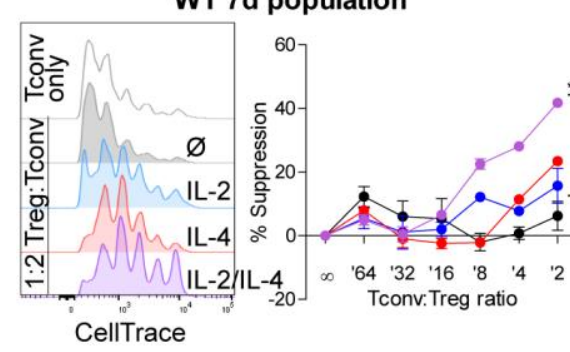

B

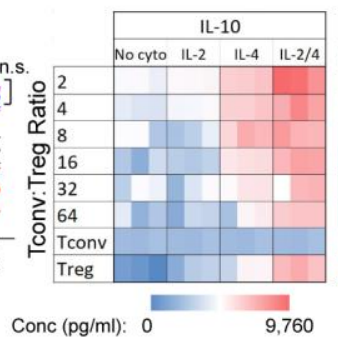

E

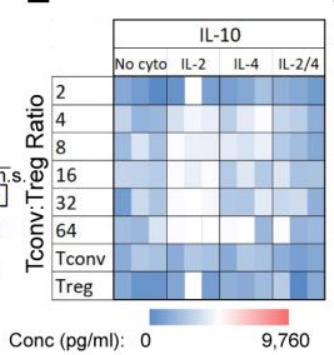

C

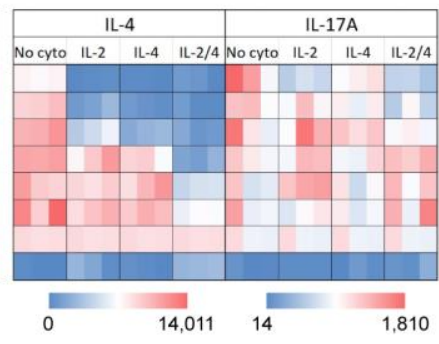

$\mathbf{F}$
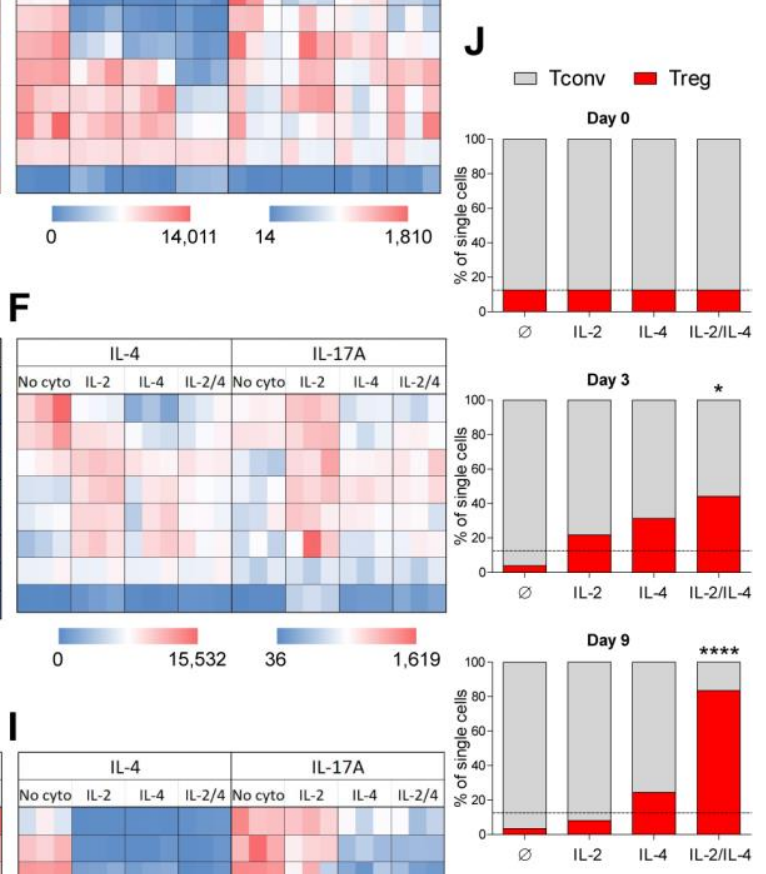
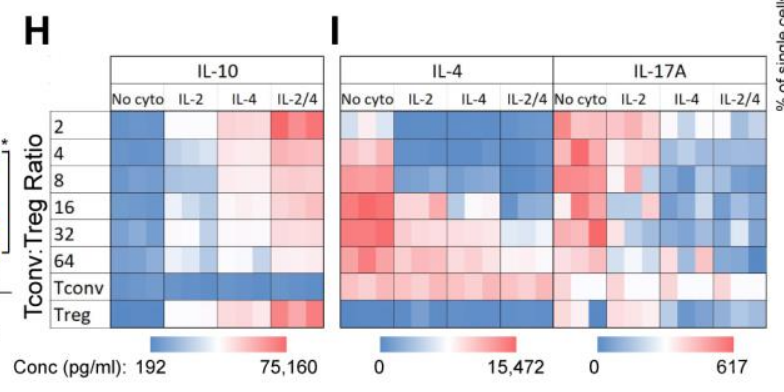

Figure 3. Combined IL-2 and IL-4 increase the suppressive ability of Tregs

752 (A-C) Proliferation (flow cytometry) and cytokine output (ELISA) of freshly isolated and Celltrace-stained Tconv co-cultured with $\alpha \mathrm{CD} 3 \varepsilon$ and the indicated ratios of WT Tregs separately and previously stimulated with $\alpha \mathrm{CD} 3 \varepsilon$ and all cytokine conditions for 3 days and normalized for cell number at the time of coculture. $\mathrm{N}=3$ for all graphs and histograms are representative. (D-F) Proliferation (flow cytometry) and cytokine production (ELISA) of freshly isolated Tconv cells cocultured with $\alpha \mathrm{CD} 3 \varepsilon$ and the indicated ratios of $\mathrm{IL}-10^{-/-}$Tregs separately and previously stimulated with $\alpha \mathrm{CD} 3 \varepsilon$ and all cytokine conditions for 3 days and normalized for cell number at the time of co-culture. $\mathrm{N}=3$ for all graphs and histograms are representative.

(G-I) Proliferation (flow cytometry) and cytokine production (ELISA) of freshly isolated Tconv cells cocultured with $\alpha \mathrm{CD} 3 \varepsilon$ and the indicated ratios of WT Tregs separately and previously stimulated with $\alpha C D 3 \varepsilon$ and all cytokine conditions for 7 days. Co-culture ratio was based on the number of Tregs prior to cytokine stimulation to incorporate their proliferation. $\mathrm{N}=3$ for all graphs and histograms are representative. over the course of time. The Tregs were purified and stimulated with $\alpha \mathrm{CD} 3 \varepsilon$ and all cytokine conditions for 3 days, washed, then placed in co-culture with freshly isolated Tconv cells at a 1:8 Treg:Tconv starting ratio (line). $\mathrm{N}=3$ 
bioRxiv preprint doi: https://doi.org/10.1101/2020.04.14.040485; this version posted April 14, 2020. The copyright holder for this preprint (which was not certified by peer review) is the author/funder, who has granted bioRxiv a license to display the preprint in perpetuity. It is made available under aCC-BY 4.0 International license.

769

For all panels, mean \pm SEM are indicated. $* p<0.05, * * * * p<0.0001$

770 
A

PSTAT3

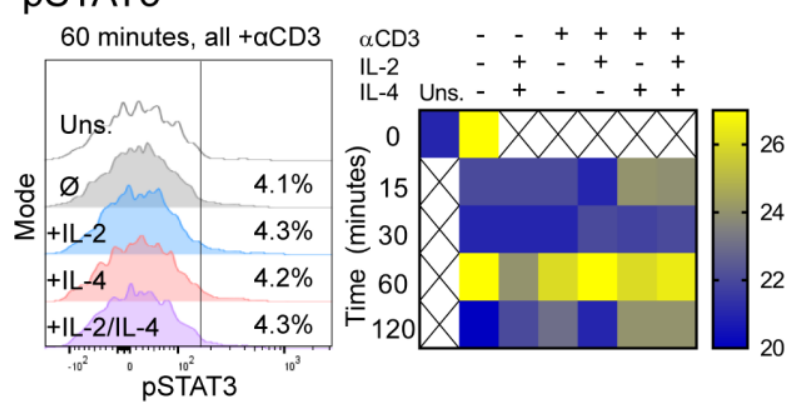

C

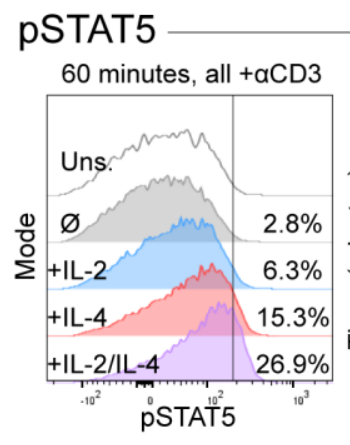

F

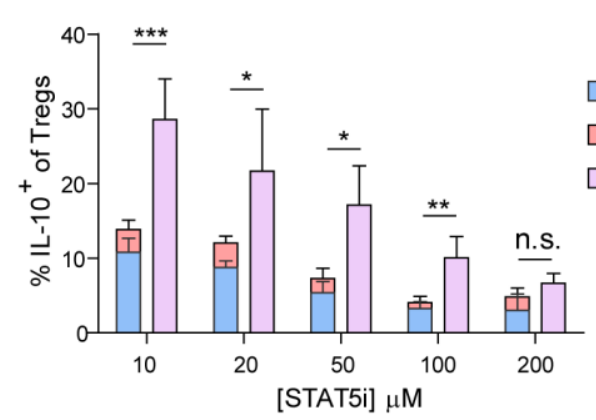

B

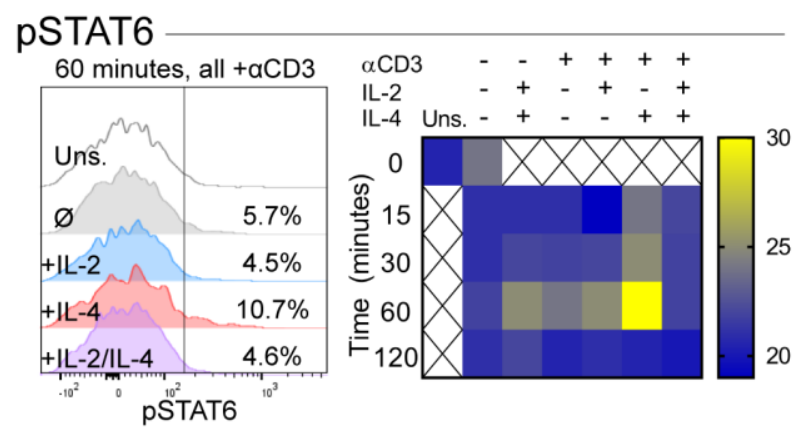

D

E
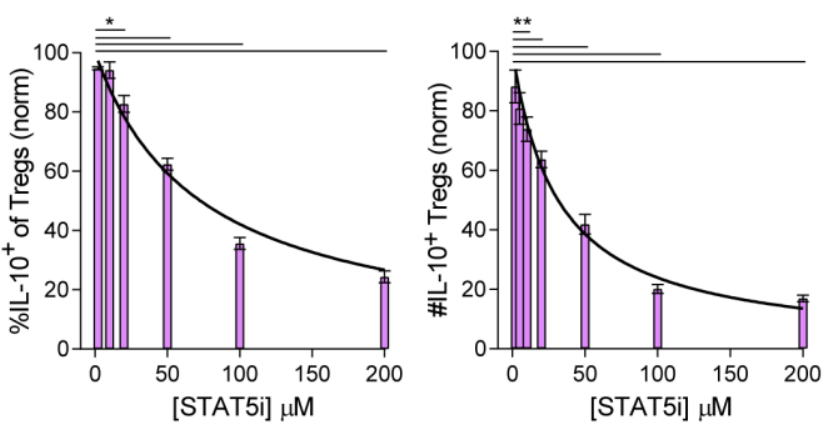

G

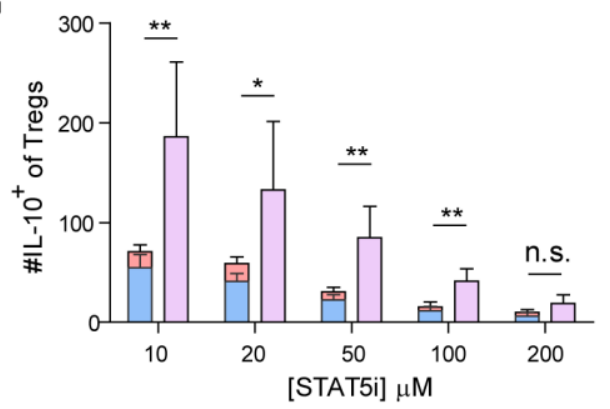

773

774

775

776

777

778

779

780

781

782

783

784

785
Figure 4. Synergistic IL-10 production and proliferation is STAT5-dependent

(A-C) pSTAT3, pSTAT6, and pSTAT5 expression of purified Tregs stimulated with the indicated culture conditions as analyzed by flow cytometry. The histograms are representative and heatmaps represent $\mathrm{N} \geq 3$ experiments.

(D-E) Treg expression of IL-10 and enumeration of IL-10+ Tregs following $\alpha$ CD3 $\varepsilon$, combined IL-2/IL-4, and STAT5i supplementation in 3 day culture, as analyzed by flow cytometry (See also Figure 4, figure supplement 1). $\mathrm{N}=3$.

(F-G) IL-10 expression in Tregs and IL-10 ${ }^{+}$Tregs counts following $\alpha \mathrm{CD} 3 \varepsilon$, all combinations of IL-2 and IL-4, and STAT5 $\mathrm{i}$ supplementation in 3 day culture, as analyzed by flow cytometry. $\mathrm{N}=3$.

For all panels, mean \pm SEM are indicated. ${ }^{*} p<0.05,{ }^{*} p<0.01, * * * p<0.001$ 


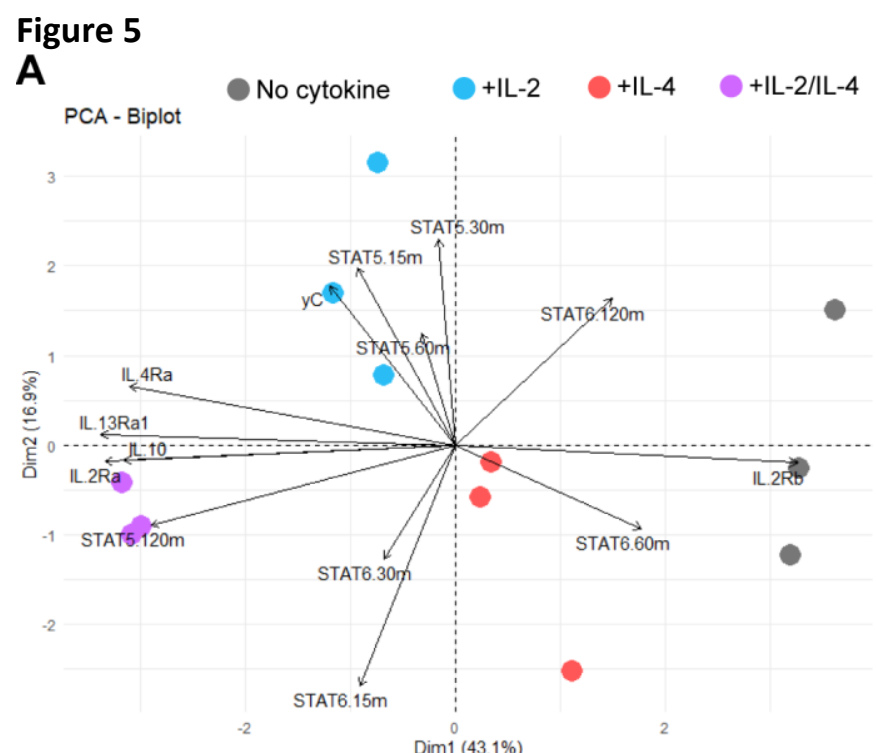

B
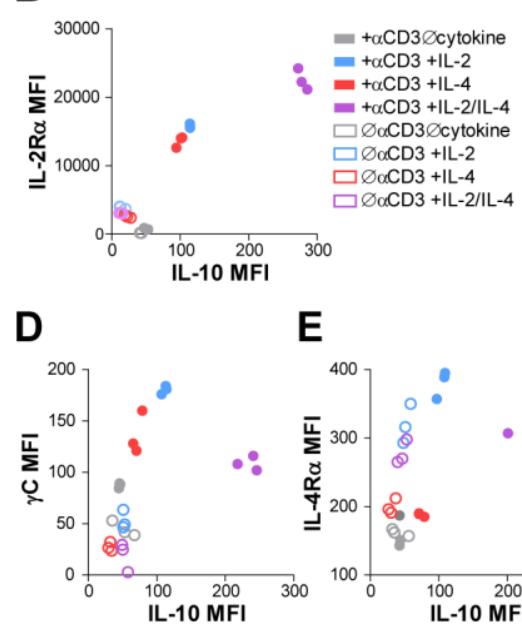

E
C

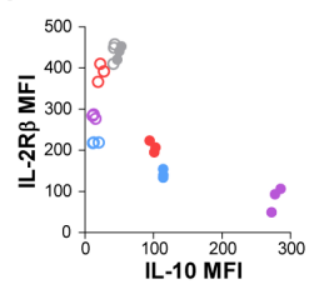

$\mathbf{F}$
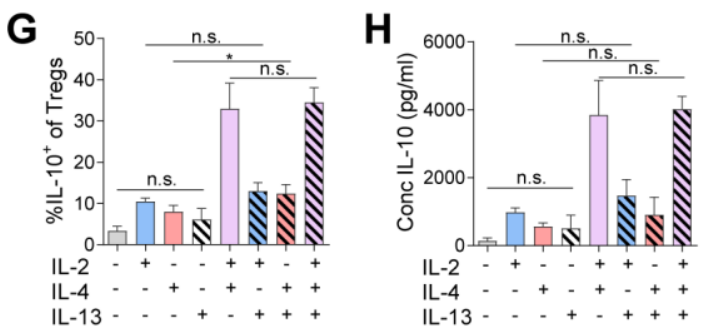

Figure 5. Combinatorial IL-2 and IL-4 signaling promotes the expression of the Type 2 IL-4 receptor (A) Expression of 14 parameters as shown in an unpaired principle component analysis of Tregs purified and stimulated with $\alpha \mathrm{CD} 3 \varepsilon$ and all cytokine conditions for 3 days unless otherwise noted in the vectors. $\mathrm{N}=3$

(B-F) Surface expression of receptor subunits that comprise the multiple forms of the IL-2R and IL-4 on purified Tregs from FoxP $3^{\text {RFP }} /$ IL-10 ${ }^{\text {GFP }}$ dual reporter mice that were stimulated for 3 days with or without $\alpha C D 3 \varepsilon$ and with all cytokine combinations. Expression of receptor subunits is plotted as a function of IL10 expression. $\mathrm{N}=3$ 
bioRxiv preprint doi: https://doi.org/10.1101/2020.04.14.040485; this version posted April 14, 2020. The copyright holder for this preprint (which was not certified by peer review) is the author/funder, who has granted bioRxiv a license to display the preprint in perpetuity. It is made available under aCC-BY 4.0 International license.

796 (G-H) Quantification of IL-10 expression and protein concentration secreted by Tregs that were purified 797 and cultured for 3 days with $\alpha \mathrm{CD} 3 \varepsilon$ and all combinations of IL-2, IL-4, and IL-13, as analyzed by flow 798 cytometry and ELISA, respectively. $\mathrm{N}=3$.

799 For all panels, mean \pm SEM are indicated. ${ }^{*} p<0.05$

800

801

802

803

804

805 


\section{Figure 6}

A

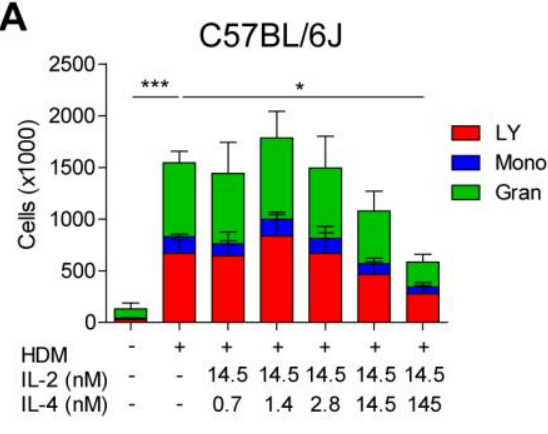

D Lung (d14)
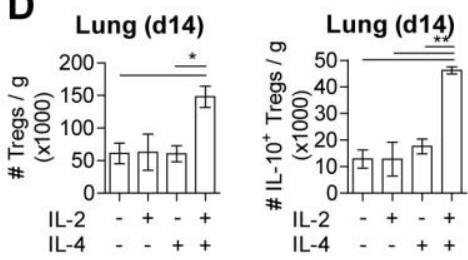

$\mathbf{B}_{\mathrm{H} \& \mathrm{E}}$

$B_{H \& E} \quad 4 x$
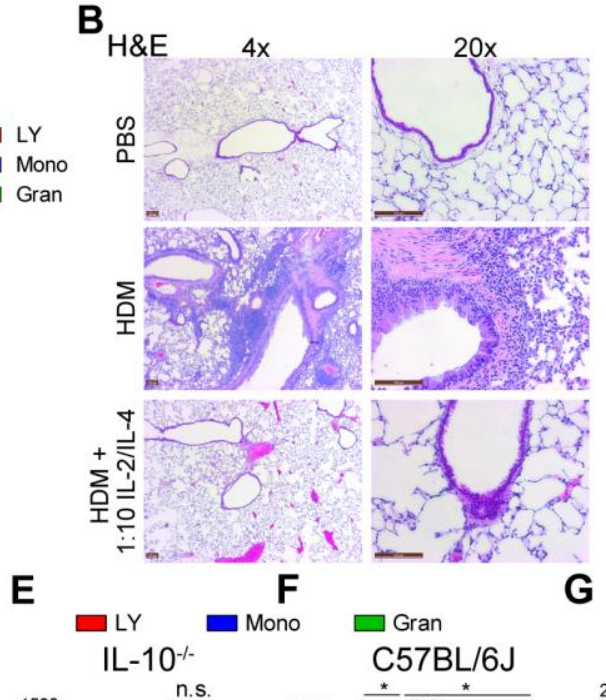

$\mathbf{G}$

$C_{\text {PAS } 4 x}$
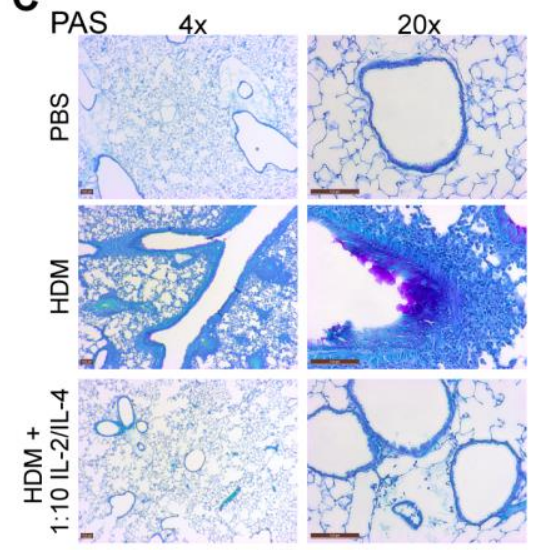

H

$\mathrm{BALB} / \mathrm{C}$
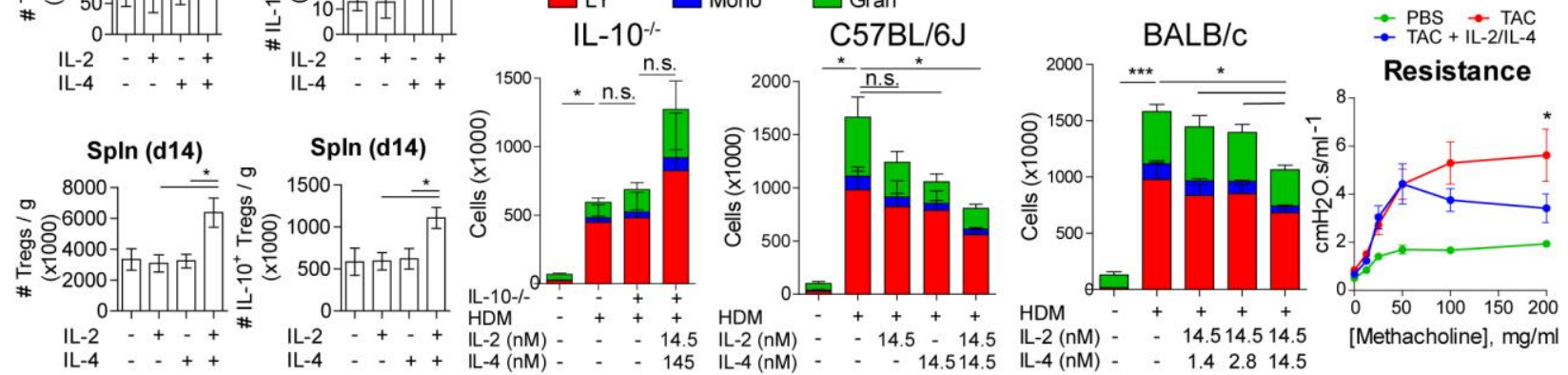

Figure 6. IL-2 and IL-4 in combination suppress the severity of HDM-induced asthma

(A-C) Analysis of HDM-induced airway inflammation in WT C57BI/6J mice by cell differentials of the Bronchial Alveolar Lavage fluid (BALf) and H\&E and PAS staining of FFPE-processed pulmonary tissues. IL-2 and IL-4 were administered i.n. on days 0-5. See also Figure Figure 6 - figure supplement $1 \mathrm{~A}$. N=6.

812 Histological images are representative.

813

814 (D) Quantification of Treg and IL- $10^{+}$Treg numbers in naïve mice following i.n. administration of combined IL-2 and IL-4 on days 0-5. Mice were harvested at day 14 for flow cytometric analysis. $\mathrm{N}=6$. (E) Assessment of airway inflammation in IL-10 ${ }^{-1-} \mathrm{C} 57 \mathrm{BI} / 6 \mathrm{~J}$ mice by cell differential analysis following i.n. challenge with HDM and i.n. administration of combined IL-2 and IL-4 in a preventative regiment in the indicated doses (See also Figure 6, figure supplement 1B). $\mathrm{N}=6$.

(F-G) Cell differential analysis of BALF quantifying the severity of HDM-induced airway inflammation in mice (C57BI/6J or BALB/C as indicated) and i.n. administration of cytokine on days 7-11 as indicated (See also Figures 6 , figure supplement $1 \mathrm{C}-\mathrm{E})$. $\mathrm{N}=6$.

(H) Airway resistance in chronic triple antigen-challenged (TAC; HDM/CRA/OVA) BALB/c mice treated or not with i.n. IL-2 and IL-4 starting in week 3 (See also Figure 6, figure supplement 2). Airway resistance was measured by Flexivent as a dose response to methacholine. $\mathrm{N} \geq 2$. For all panels, mean \pm SEM are indicated. ${ }^{*} p<0.05,{ }^{* *} p<0.01,{ }^{* * *} p<0.001$ 


\section{Figure 7}

A

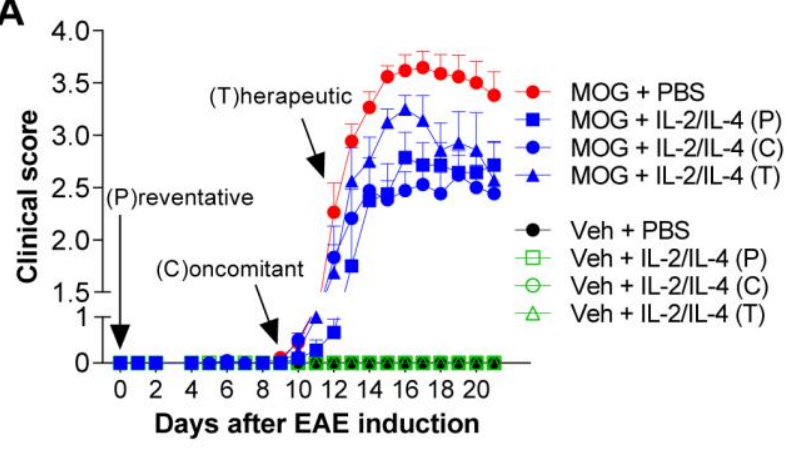

B
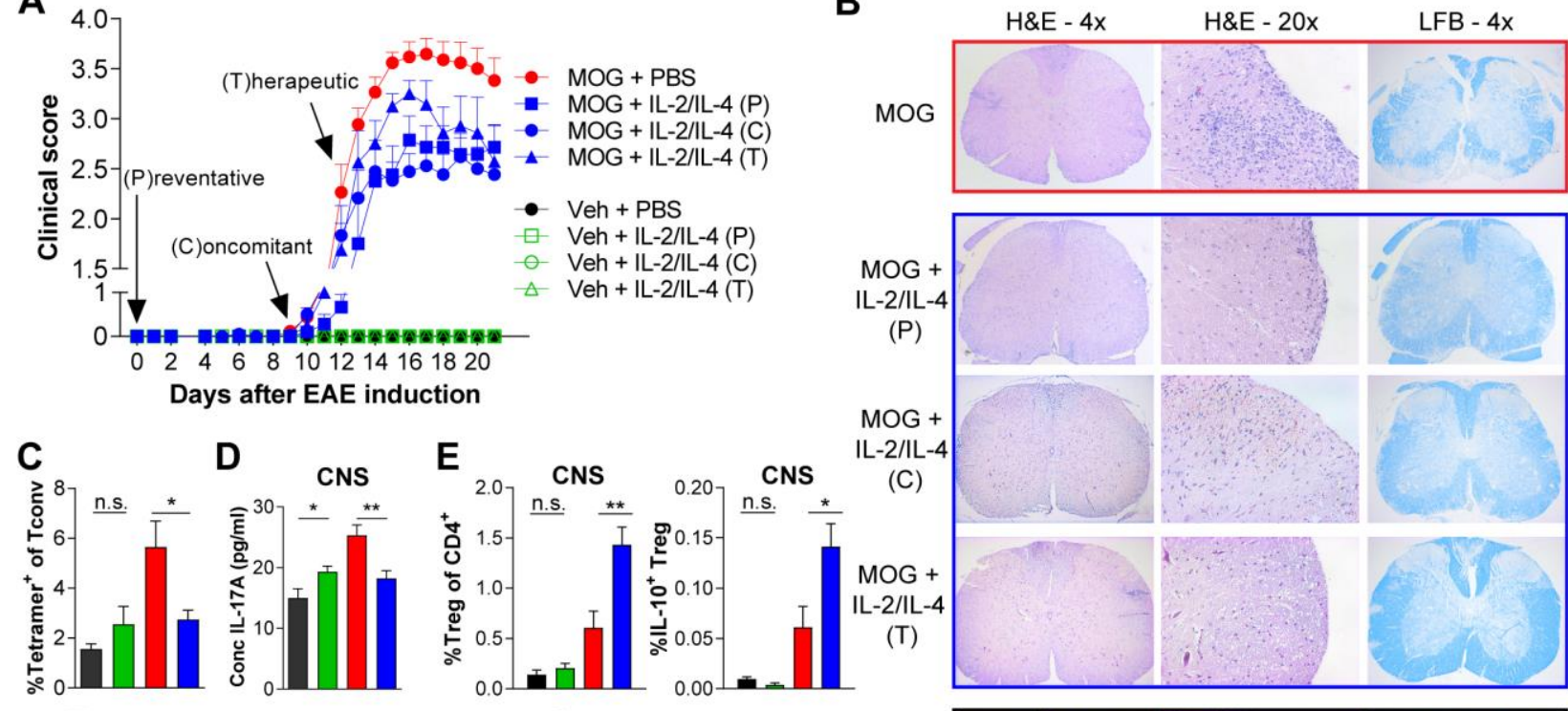

$\mathbf{F}$

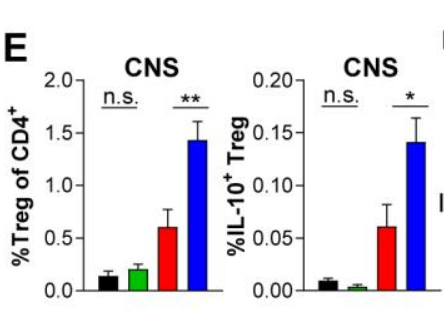

(C)

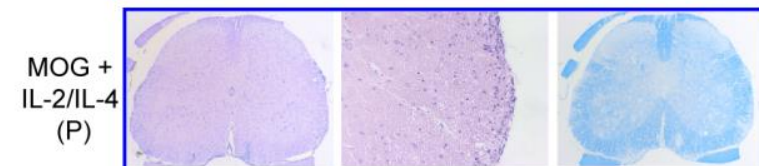

MOG + IL-2/IL-4

( $\mathrm{T})$

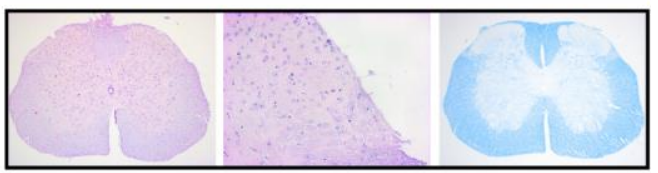

H

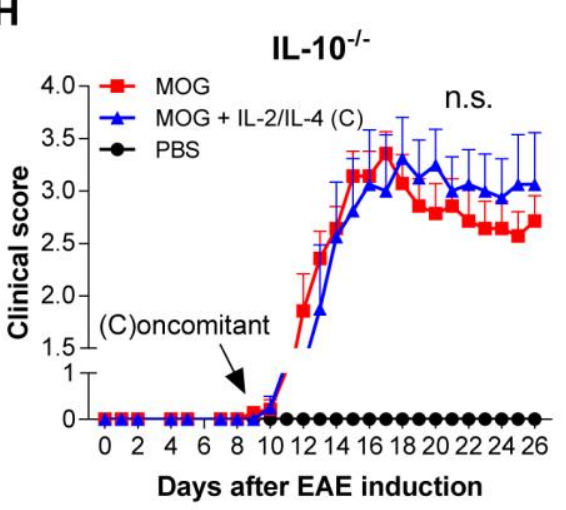

Veh

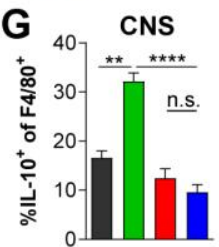

I

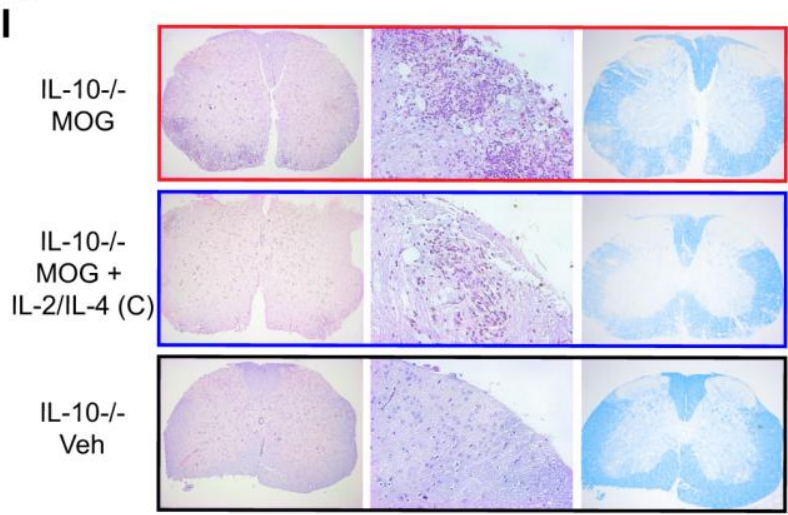

\section{$\mathbf{J}$}

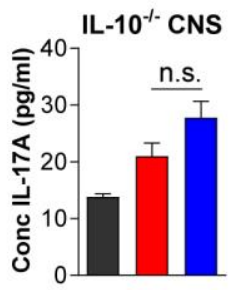

Figure 7. Combined IL-2 and IL-4 reduces the severity of EAE

(A-B) EAE clinical score and FFPE-processed spinal cords sectioned and stained with H\&E and LFB from mice challenged with MOG peptide with and without combined cytokines (See also Figure 7 - figure supplement 1). Histological images represent a mouse of the mean disease score of each condition (See also Figure 7 - figure supplement $1 \mathrm{C}$ ). $\mathrm{N}=10-12$ for $\mathrm{EAE}$ mice; $\mathrm{N}=4$ for vehicle control mice. (C) Analysis of MOG-loaded MHCII tetramer positive Tconv cells in the CNS as determined by flow cytometry. $\mathrm{N}=3$

837 (D) Quantification of IL-17A secreted by CNS leukocytes isolated by Percoll gradient and incubated 838 overnight in media, as quantified by ELISA. $\mathrm{N}=3$

839 (E-F) Analysis of Treg abundance and IL-10-expression in Tregs isolated from the CNS or spleen of 840 FoxP3 ${ }^{\text {RFP }} /$ IL- $10^{\text {GFP }}$ mice in the EAE trials by flow cytometry. $\mathrm{N}=3$ 
bioRxiv preprint doi: https://doi.org/10.1101/2020.04.14.040485; this version posted April 14, 2020. The copyright holder for this preprint (which was not certified by peer review) is the author/funder, who has granted bioRxiv a license to display the preprint in perpetuity. It is made available under aCC-BY 4.0 International license.

841 (G) Analysis of IL-10 expression of $\mathrm{F} 4 / 80^{+} \mathrm{CNS}$ macrophages from FoxP3 ${ }^{\mathrm{RFP}} / \mathrm{IL}-10^{\mathrm{GFP}}$ mice in the EAE trials 842 by flow cytometry. $\mathrm{N}=3$

$843(\mathrm{H}-\mathrm{I})$ Assessment of EAE severity in IL-10/- mice as quantified by daily clinical scoring and FFPE-processing 844 of spinal cords stained with H\&E and LFB. Images represent a mouse of the mean disease score of each 845 condition. $\mathrm{N}=7-8$ for $\mathrm{EAE}$ mice; $\mathrm{N}=4$ for negative control mice for disease

846 (J) Quantification of IL-17A secreted by CNS leukocytes of IL-10 $1-$ mice isolated by Percoll gradient and 847 incubated overnight in media, as quantified by ELISA. $\mathrm{N}=3$

848 For all panels, mean \pm SEM are indicated. ${ }^{*} p<0.05, * * p<0.01, * * * * p<0.0001$ 


\section{Supplementary Materials}

853

854

Figure 1, figure supplement 1

A

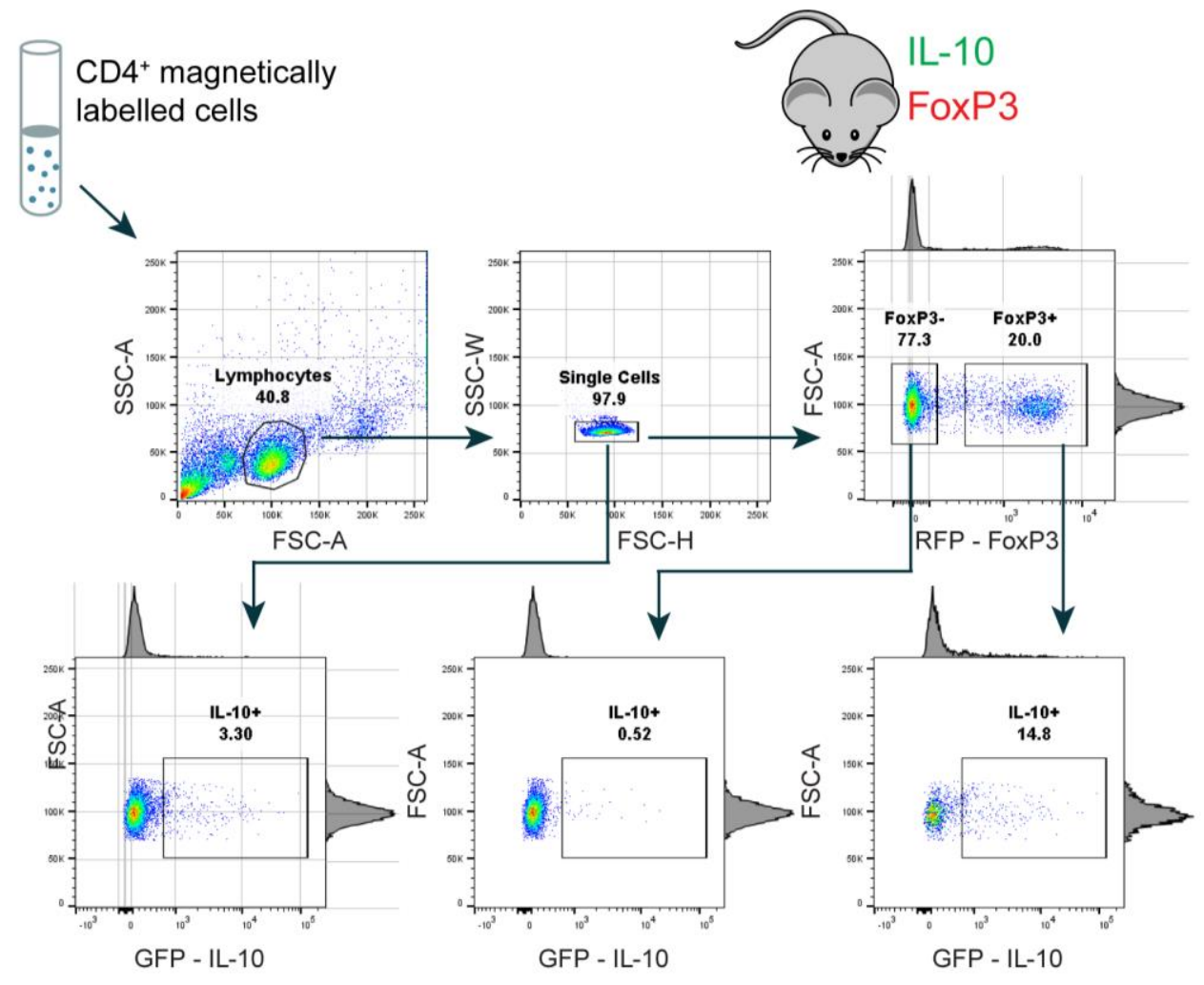

B

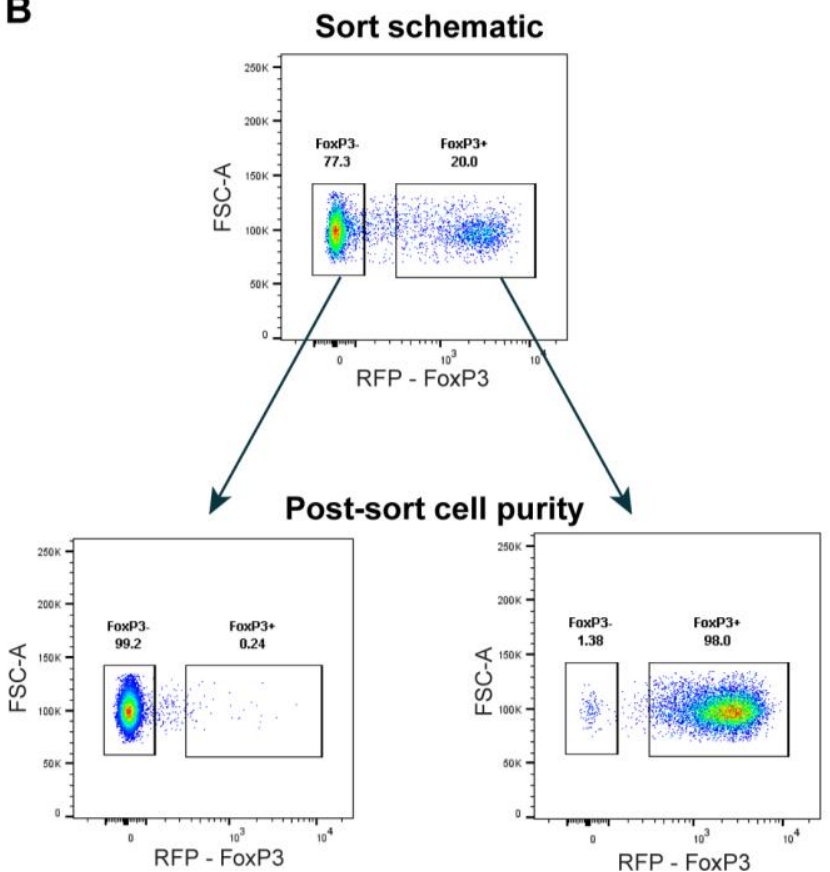

Figure 1, figure supplement 1. Cell sorting gating strategy and post-sort purity 
bioRxiv preprint doi: https://doi.org/10.1101/2020.04.14.040485; this version posted April 14, 2020. The copyright holder for this preprint (which was not certified by peer review) is the author/funder, who has granted bioRxiv a license to display the preprint in perpetuity. It is made available under aCC-BY 4.0 International license.

857 (A) Gating strategy for flow cytometry sorting and analyzing IL-10 ${ }^{+/-}$Tregs from FoxP3 ${ }^{\text {RFP }} /$ IL-10 ${ }^{\text {GFP }}$ dual858 reporter mice. Freshly harvested spleens underwent $\mathrm{CD} 4^{+}$magnetic bead selection prior to FACS.

859 Related to Figure 1. (B) Sorted cells were immediately analyzed by flow cytometry to determine purity.

860 Related to Figure 1.

861

862 
Figure 1, figure supplement 2

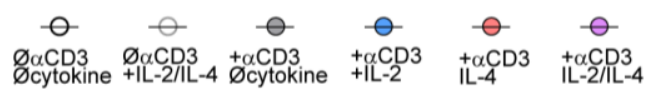

A

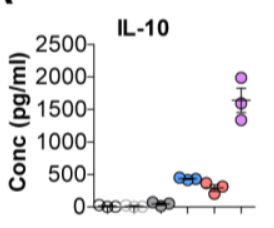

C
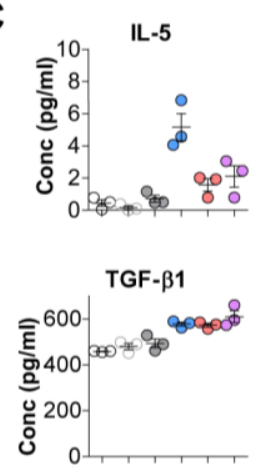

B
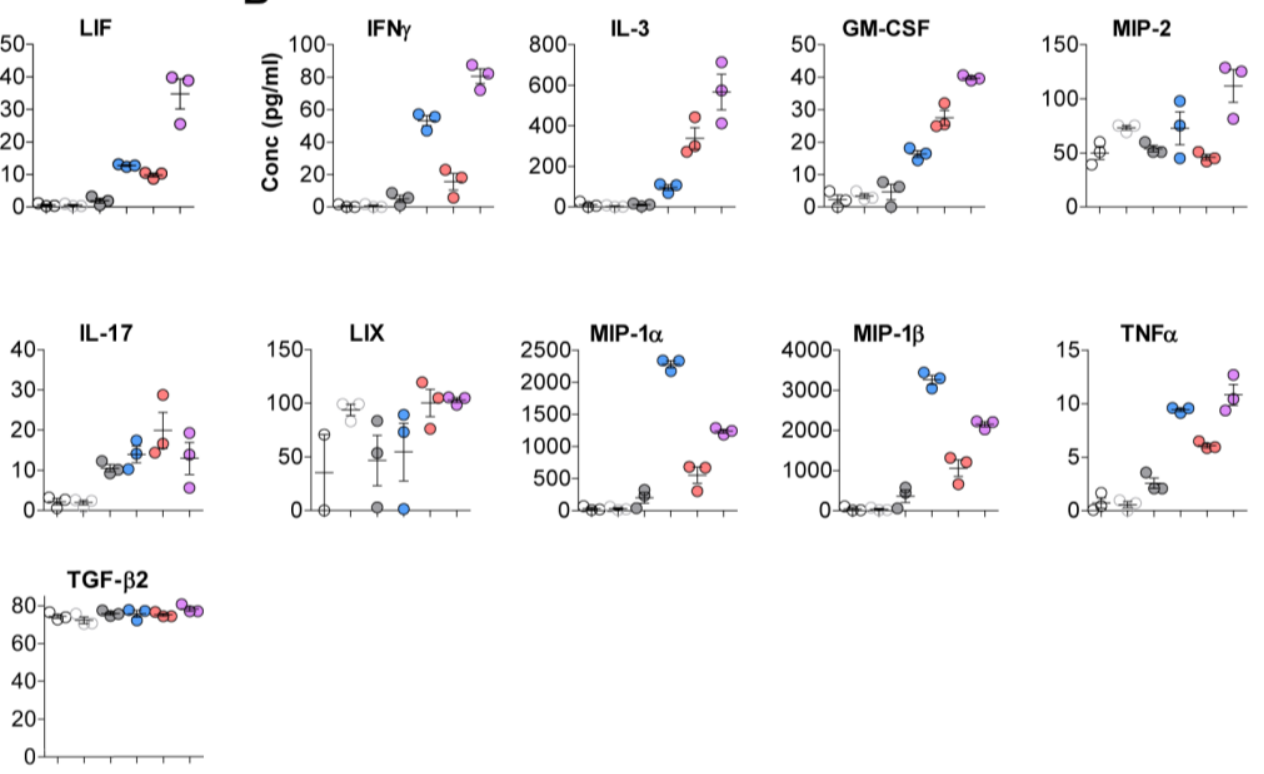

D
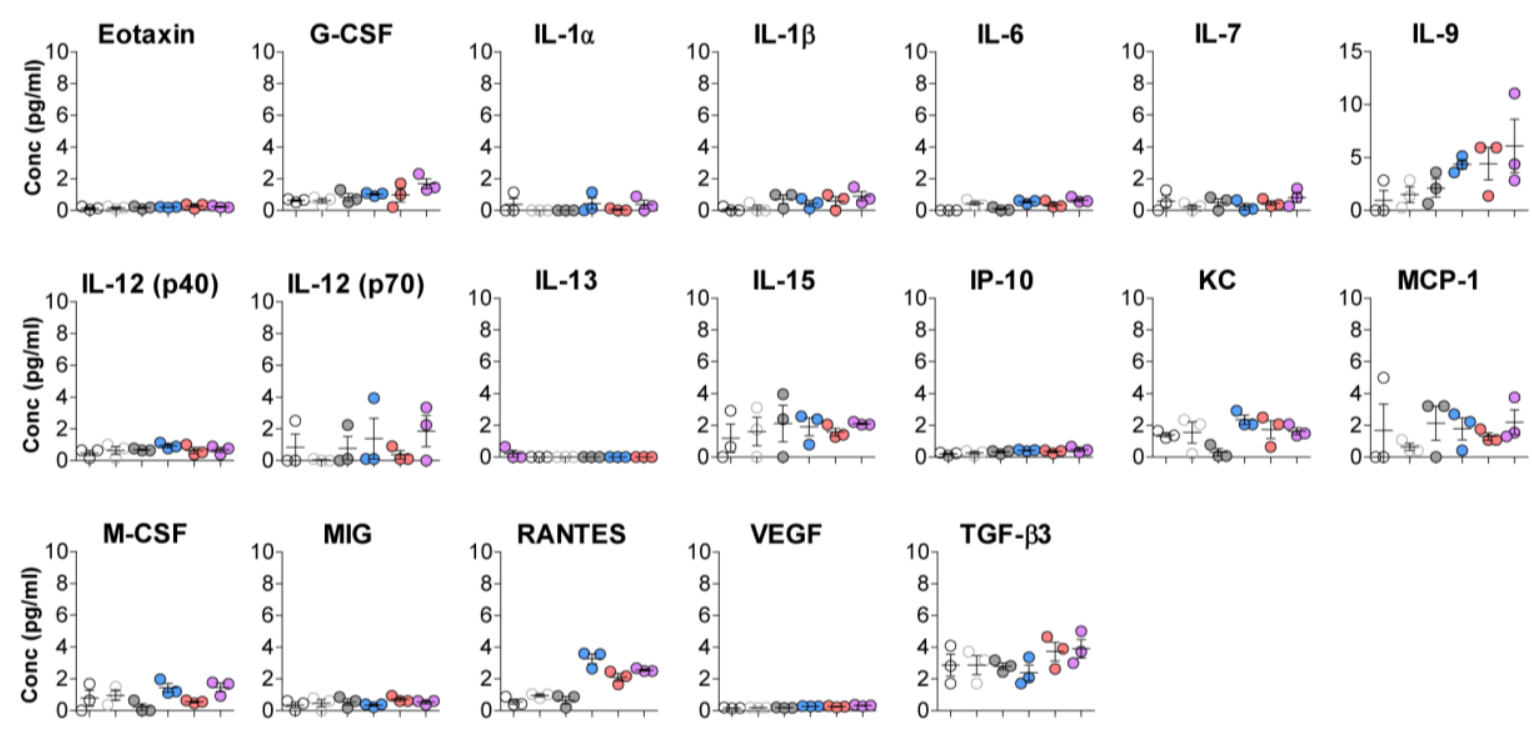

Figure 1, figure supplement 2. IL-10 is the only synergistic and robust analyte produced by Tregs following combinatorial cytokine stimulation

867 31-plex Luminex analysis of cytokines secreted into the media following the designated stimulation conditions grouped in cytokines that (A) synergistically increase with combinatorial cytokine stimulation, (B) are summative, (C) show no change compared to single cytokine stimulation, (D) are not highly expressed. Error bars indicate mean $\pm \mathrm{SEM}$. $\mathrm{N}=3$. Related to Figure $1 \mathrm{~J}$. 
bioRxiv preprint doi: https://doi.org/10.1101/2020.04.14.040485; this version posted April 14, 2020. The copyright holder for this preprint (which was not certified by peer review) is the author/funder, who has granted bioRxiv a license to display the preprint in perpetuity. It is made available under aCC-BY 4.0 International license.

\section{Figure 1, figure supplement 3}

A

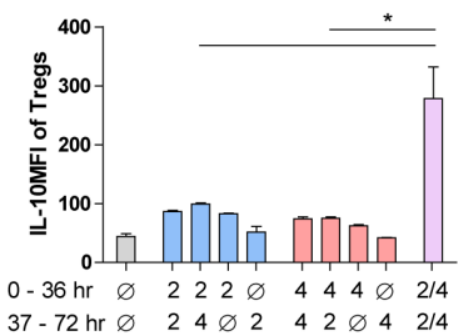

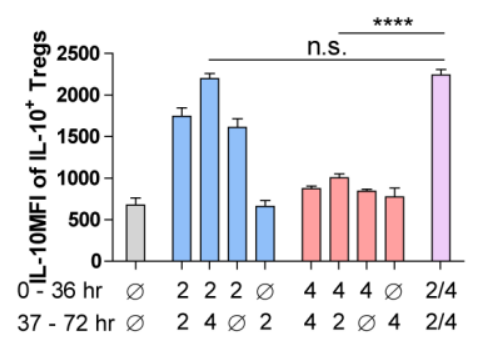

B

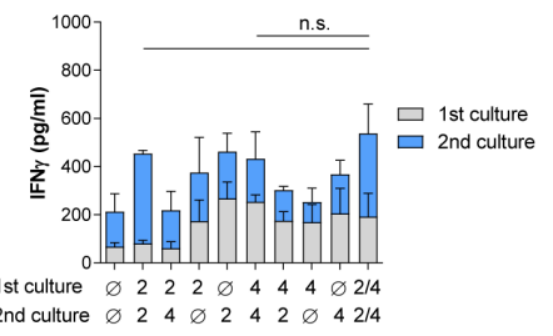

C

Day 0

Day 3

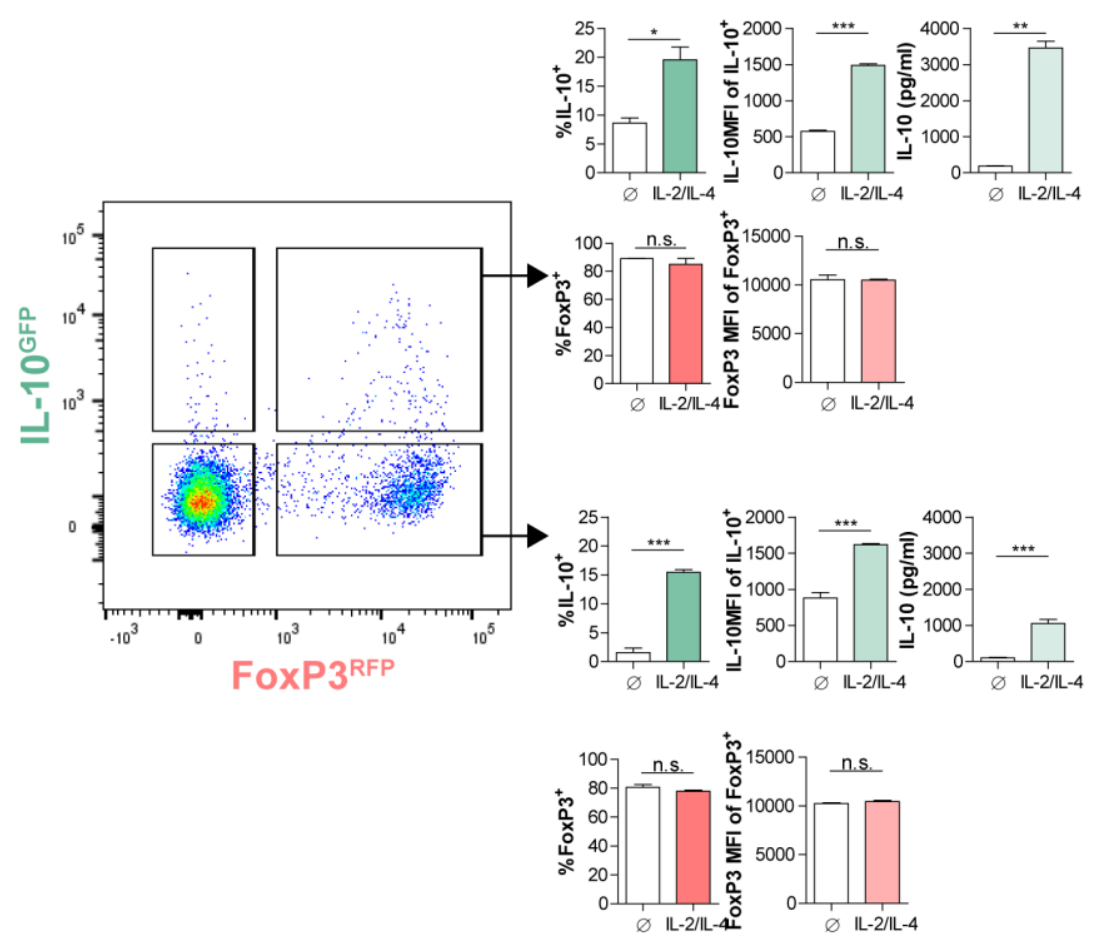

D
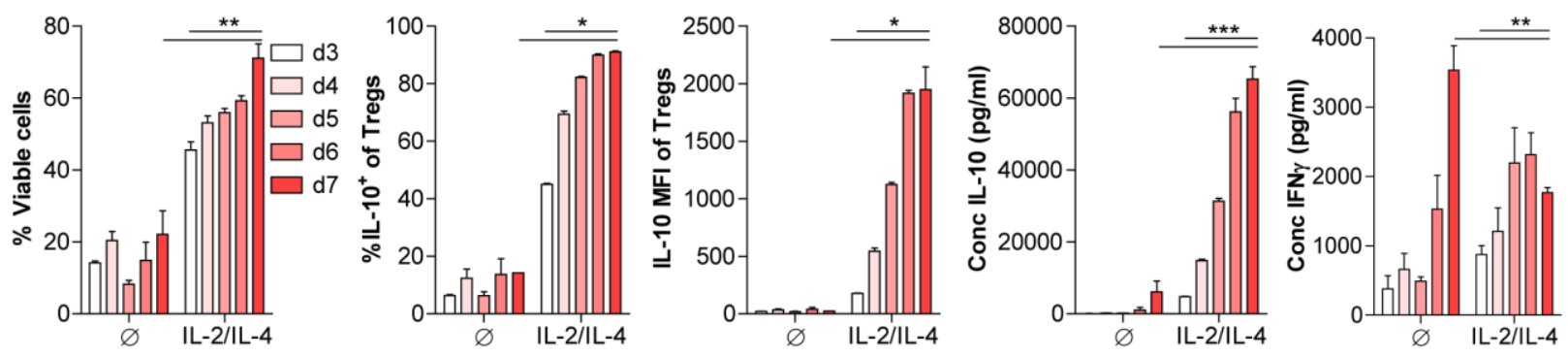
bioRxiv preprint doi: https://doi.org/10.1101/2020.04.14.040485; this version posted April 14, 2020. The copyright holder for this preprint (which was not certified by peer review) is the author/funder, who has granted bioRxiv a license to display the preprint in perpetuity. It is made available under aCC-BY 4.0 International license.

873 Figure 1, figure supplement 3. Dynamics of combinatorial cytokine stimulation of Tregs over time

874 (A) IL-10 expression of purified Tregs stimulated for 36 hours in culture with the first set of conditions, 875 washed, and subsequently stimulated for another 36 hours in culture with the second set of conditions, 876 as analyzed by flow cytometry. All samples received $\alpha \mathrm{CD} 3 \varepsilon$ activation. $\mathrm{N}=3$. Related to Figure $1 \mathrm{H}(\mathrm{B})$ 877 Quantification of IFNy secretion following the culture conditions as detailed above, as analyzed by ELISA. $878 \mathrm{~N}=3$. Related to Figure 1I. (C) IL-10 and FoxP3 expression of purified IL-10 $10^{+-}$Tregs that underwent culture 879 with $\alpha \mathrm{CD} 3 \varepsilon$ and combined IL-2/IL-4for 3 days, as analyzed by flow cytometry. $\mathrm{N}=3$. Related to Figure $1 \mathrm{~L}$. 880 (D) Analysis of viability and IL-10 expression of purified Tregs cultured with $\alpha$ CD3 $\varepsilon$ and combined IL-2/IL8814 for 3-7 days, as analyzed by flow cytometry. IL-10 and IFN $\gamma$ content in the culture media was analyzed 882 by ELISA.

883 For all panels, mean \pm SEM are indicated. ${ }^{*} p<0.05,{ }^{* *} p<0.01,{ }^{* * *} p<0.001,{ }^{* * * *} p<0.0001$ 
Figure 4, figure supplement 1

A

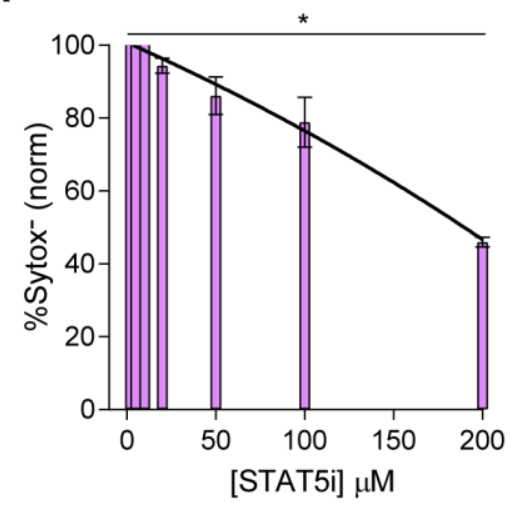

C

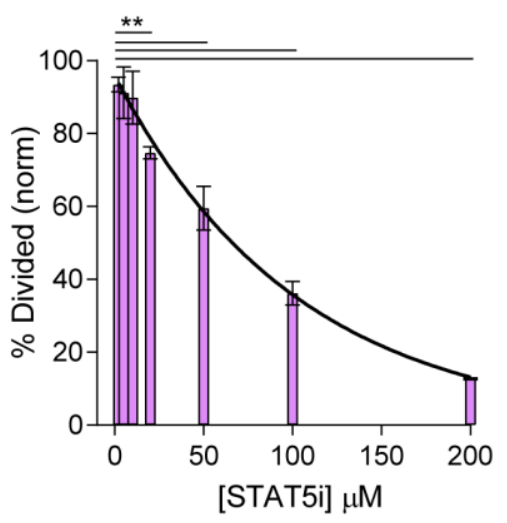

E

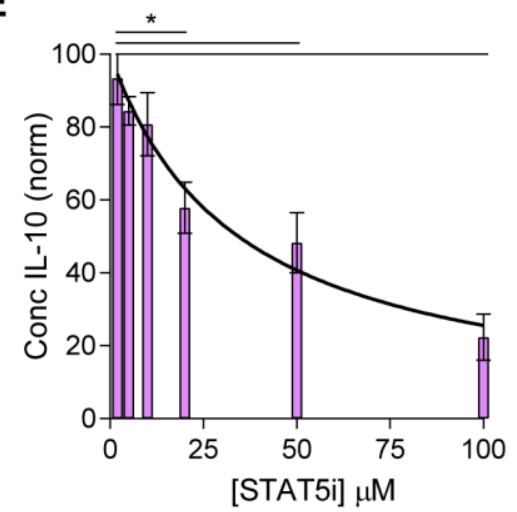

B

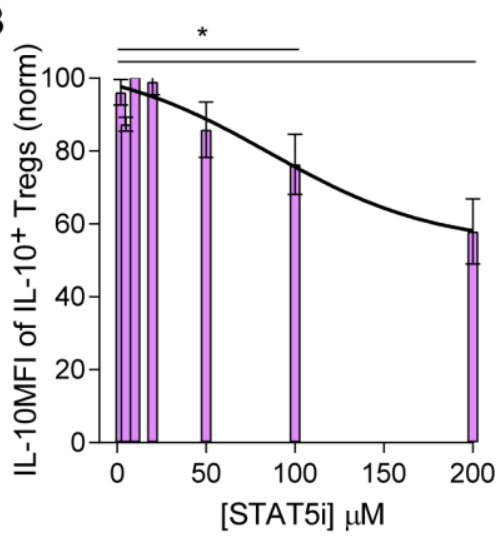

D

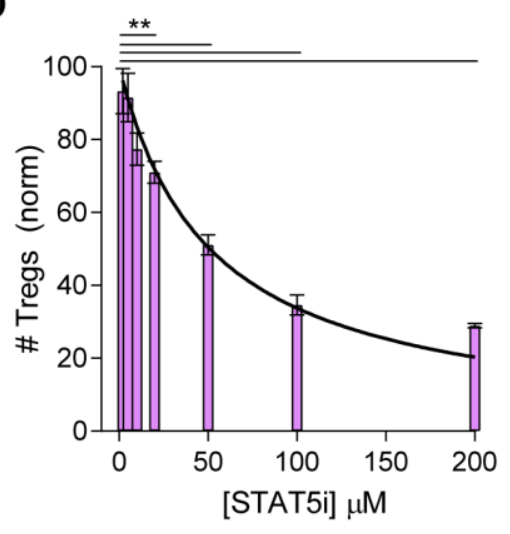

$\mathbf{F}$

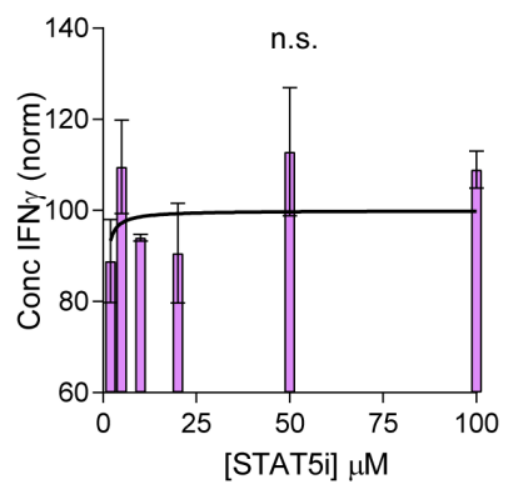

Figure 4, figure supplement 1. The IL-10 and proliferative response following combined IL-2 and IL-4 stimulation is dependent on STAT5 signaling (A-D) Analysis of viability (Sytox Red), IL-10 expression, and proliferation of purified Tregs that were cultured with $\alpha \mathrm{CD} 3 \varepsilon$, combined IL-2 and IL-4, and STAT5i (resuspended in DMSO) in the indicated concentrations for 3 days, as analyzed by flow cytometry. The values are normalized to Tregs cultured with the same stimulation conditions but with DMSO only and no STAT5i. (E-F) Quantification of IL-10 and IFN $\gamma$ secreted by the cells in the culture setup above as analyzed by ELISA. N=3. Related to Figure 4. 
bioRxiv preprint doi: https://doi.org/10.1101/2020.04.14.040485; this version posted April 14, 2020. The copyright holder for this preprint (which was not certified by peer review) is the author/funder, who has granted bioRxiv a license to display the preprint in perpetuity. It is made available under aCC-BY 4.0 International license.

Figure 6, figure supplement 1

A

Acute preventative (HDM)

i.n. challenge $\overbrace{\mathrm{L}-2 / \mathrm{L}-4 \mathrm{LL}-2 / \mathrm{LL}-4 \mathrm{IL}-2 / \mathrm{LL}-4 \mathrm{IL}-2 / \mathrm{LL}-4 \mathrm{IL}-2 / \mathrm{LL}-4}^{\text {i.n. therapy }}$

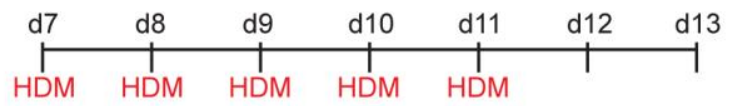

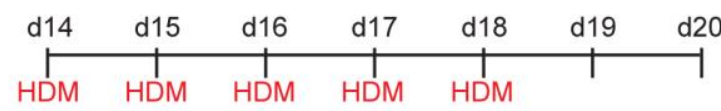

d21

Harvest
B

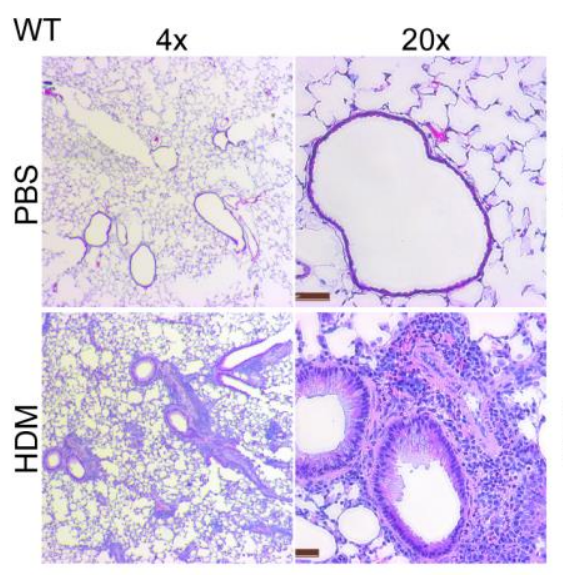

IL-10-/-

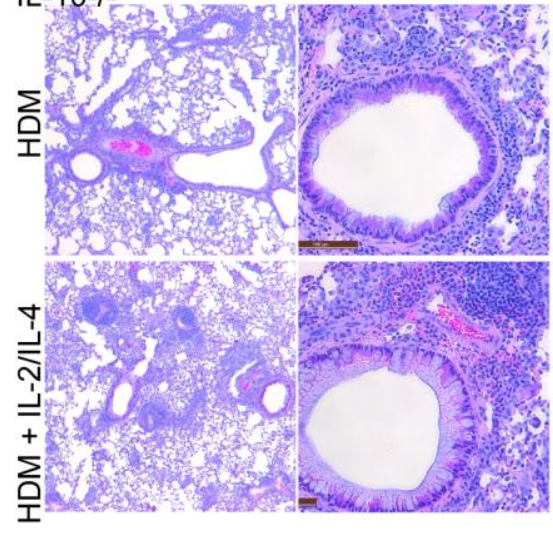

899

900

901 and is dependent on IL-10
C Acute therapeutic (HDM)
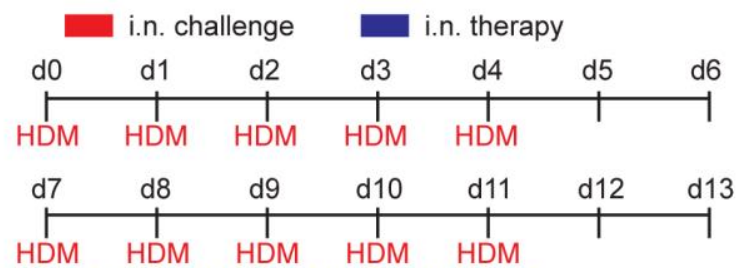

IL-2/IL-4 IL-2/IL-4 IL-2/IL-4 IL-2/IL-4 IL-2/IL-4

d14

Harvest
D
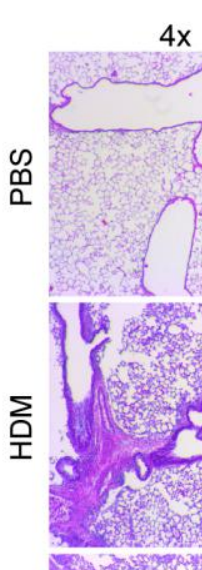

C57BL/6J
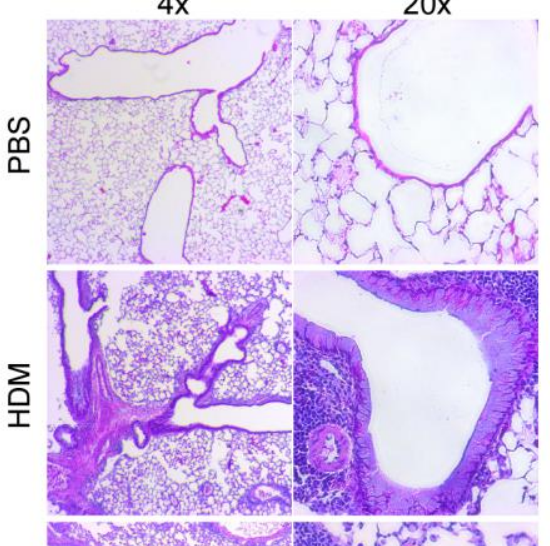

E
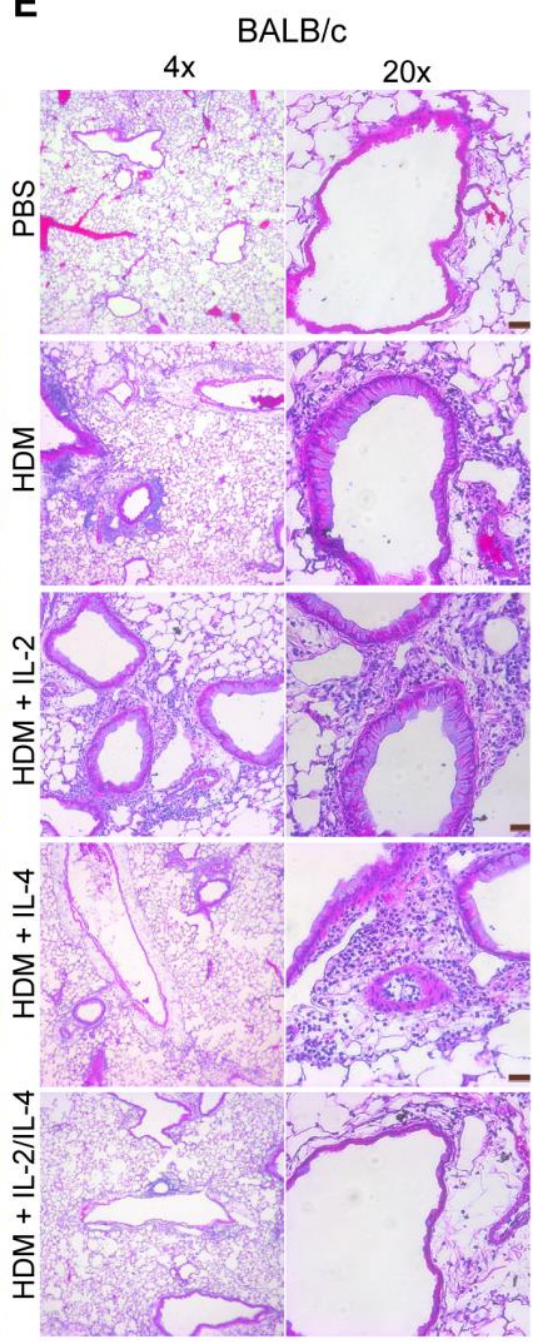

Figure 6, figure supplement 6. Administration of IL-2 and IL-4 suppresses HDM-induced acute asthma 
bioRxiv preprint doi: https://doi.org/10.1101/2020.04.14.040485; this version posted April 14, 2020. The copyright holder for this preprint (which was not certified by peer review) is the author/funder, who has granted bioRxiv a license to display the preprint in perpetuity. It is made available under aCC-BY 4.0 International license.

902

903

904

905

906

907

908

909

910

911

(A) Challenge and treatment schedule for preventative HDM-induced acute asthma trial in Figures 6A and 6E. IL-2/IL-4 was delivered i.n. for 5 consecutive days (d0-5), followed by 2 weeks of HDM (or PBS) challenge through i.n. challenge (d7-11, d14-d18). (B) Representative H\&E histology for Figure 6E. N=6 mice. (C) Challenge and treatment schedule for therapeutic HDM-induced acute asthma trial in Figure 6F. Mice were challenged with HDM (or PBS) for 2 weeks (d7-11, d14-18). IL-2/IL-4 was administered i.n. for 5 days starting on $d 7$. (D) The severity of pulmonary disease was analyzed by FFPE tissues that underwent H\&E staining and imaging at 4x and 20x. N=6 mice. Representative H\&E histology for Figure 6F. (E) Pulmonary disease was analyzed by FFPE tissues that underwent sectioning, H\&E staining, and imaging at 4x and 20x. N=6 mice. Representative H\&E histology for Figure 6G. 
bioRxiv preprint doi: https://doi.org/10.1101/2020.04.14.040485; this version posted April 14, 2020. The copyright holder for this preprint (which was not certified by peer review) is the author/funder, who has granted bioRxiv a license to display the preprint in perpetuity. It is made available under aCC-BY 4.0 International license.

\section{Figure 6, figure supplement 2}

\section{A}

\section{Chronic therapeutic (TAC)}

i.p. sensitization $\square$ i.n. challenge $\square$ i.n. therapy

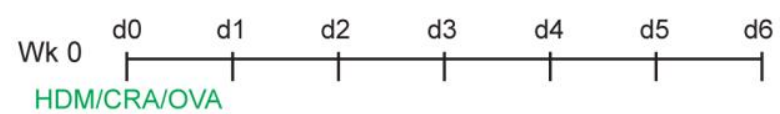

Wk1

Wh $2 \underset{\text { HDM/CRA/OVA }}{\mathrm{d} 14} \mathrm{~d} 15 \quad \mathrm{~d} 16 \quad \mathrm{~d} 17 \quad \mathrm{~d} 18 \quad \mathrm{~d} 19 \quad \mathrm{~d} 20$

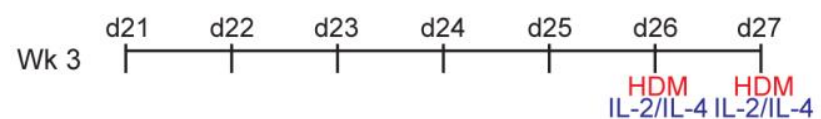

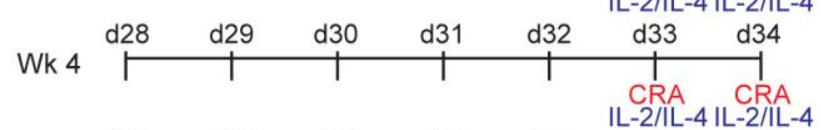

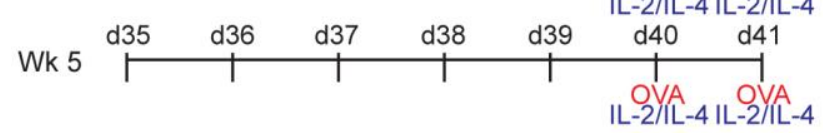

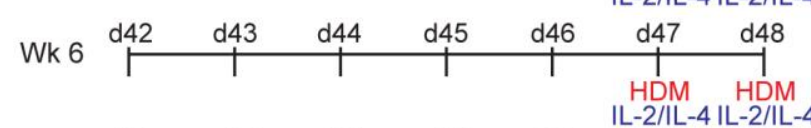

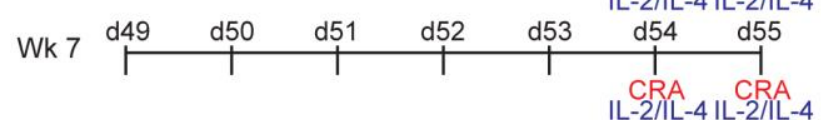

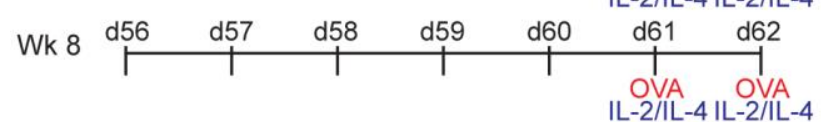

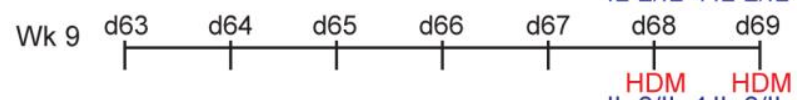
Wk $10 \underset{\text { Harvest }}{\mathrm{d} 70}$
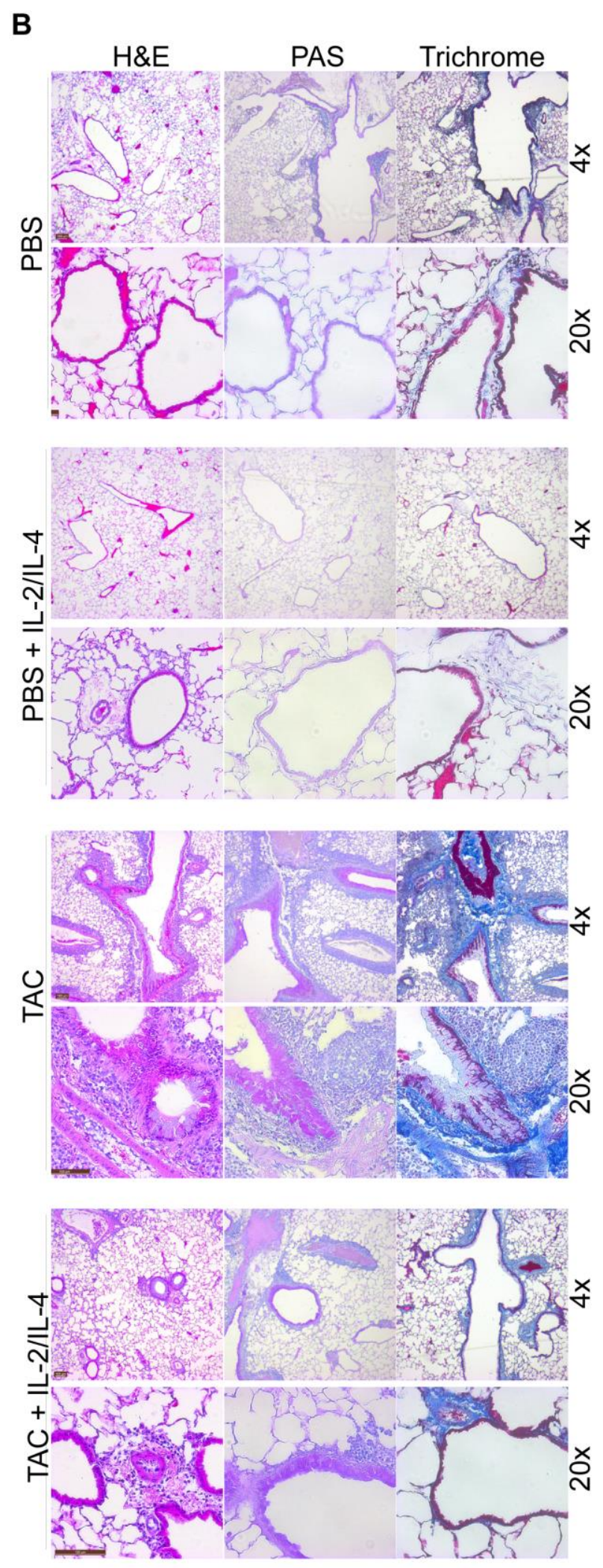
bioRxiv preprint doi: https://doi.org/10.1101/2020.04.14.040485; this version posted April 14, 2020. The copyright holder for this preprint (which was not certified by peer review) is the author/funder, who has granted bioRxiv a license to display the preprint in perpetuity. It is made available under aCC-BY 4.0 International license.

914 Figure 6, figure supplement 2. Administration of IL-2 and IL-4 therapeutically suppresses TAC-induced 915 chronic asthma

916 (A) Challenge and treatment schedule for therapeutic TAC-induced chronic asthma trial in Figure $6 \mathrm{H}$.

917 Mice were sensitized to the triple antigens once weekly for the first three weeks. Afterwards, all

918 challenges and treatments were delivered i.n. Mice received HDM, CRA, and OVA in a rotating weekly

919 schedule, and also received IL-2 and IL-4 in combination following the challenge. (B) Pulmonary disease

920 severity was analyzed by FFPE tissues that underwent H\&E, PAS, Trichrome staining and imaged at $4 x$

921 and 20x. $\mathrm{N} \leq 5$. Representative H\&E histology for Figure $6 \mathrm{H}$. 


\section{Figure 7, figure supplement 1}

A

EAE Trial

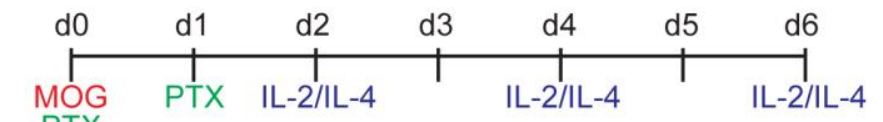

IL-2/IL-4P
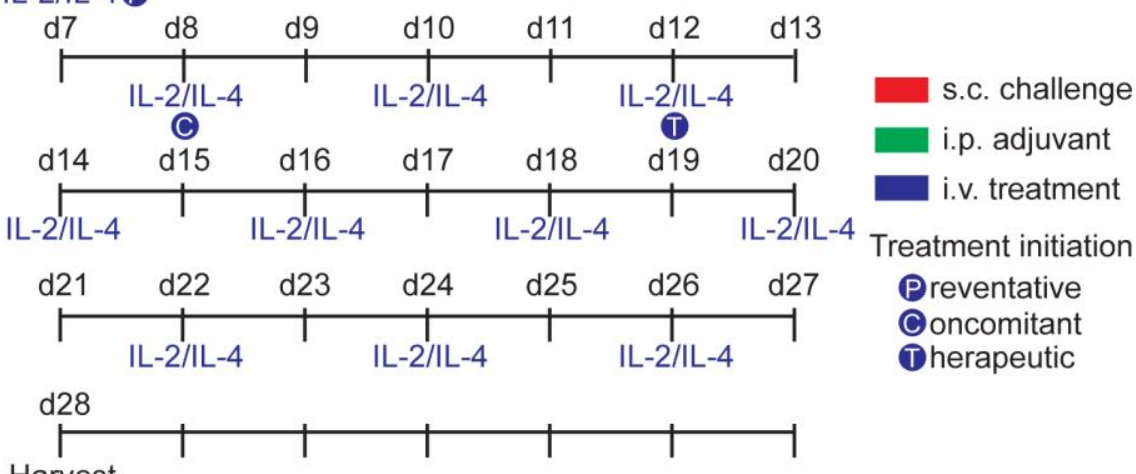

Harvest

B

Table of $P$-values for Figure 7A

\begin{tabular}{|l|r|r|r|}
\hline Day & $\begin{array}{c}\text { MOG + PBS vs } \\
\text { Preventative Tx }\end{array}$ & $\begin{array}{c}\text { MOG + PBS vs } \\
\text { Concomitant tx }\end{array}$ & $\begin{array}{c}\text { MOG + PBS vs } \\
\text { Therapeutic tx }\end{array}$ \\
\hline 11 & 0.0307 & 0.2215 & 0.2539 \\
\hline 12 & 0.0014 & 0.8175 & 0.5854 \\
\hline 13 & 0.0079 & 0.9653 & 0.6730 \\
\hline 14 & 0.0186 & 0.3031 & 0.2140 \\
\hline 15 & 0.0001 & 0.0299 & 0.2549 \\
\hline 16 & 0.0075 & 0.0085 & 0.0683 \\
\hline 17 & 0.0027 & 0.0000 & 0.0207 \\
\hline 18 & 0.0118 & 0.0000 & 0.1407 \\
\hline 19 & 0.0155 & 0.0000 & 0.0887 \\
\hline 20 & 0.0205 & 0.0002 & 0.0381 \\
\hline 21 & 0.0921 & 0.0020 & 0.1050 \\
\hline
\end{tabular}

C

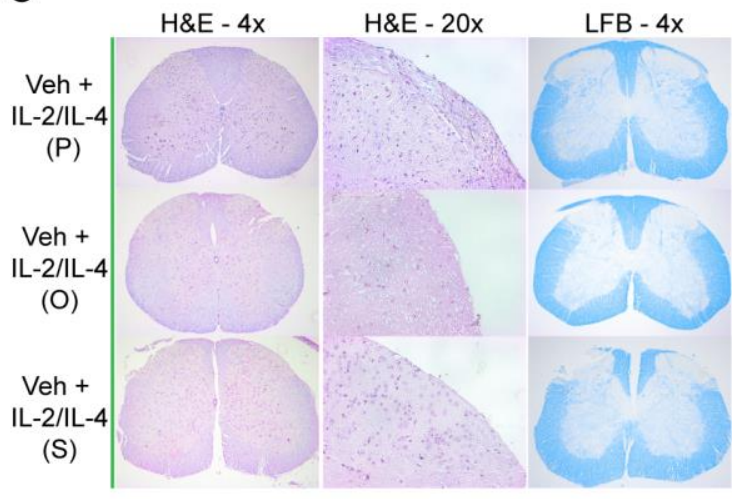

923

924

925

926

927

928

929

930

931

932

933

934

935

Figure 7, figure supplement 1. Combined IL-2 and IL-4 suppressed the severity of EAE in Preventative, Concomitant, and Therapeutic dosing regiment

(A) EAE was induced in mice with s.c. injections of MOG emulsified in Complete Freund's Adjuvant (CFA) and d0. Pertussis toxin (PTX) was injected i.p. on days 0 and 1. Negative control mice received CFA only with PTX. The combinatorial cytokines were delivered i.v., with the Preventative regimen beginning on $\mathrm{d} 0$, Concomitant regimen on $\mathrm{d} 8$, and Therapeutic regimen on $\mathrm{d} 12$. Mice that did not receive the combinatorial cytokines received i.v. PBS as the vehicle control. Related to Figure 7. (B) Table of P values for Figure 7A. Red font represents $P \leq 0.05$. Black font represents $P>0.05$. $N=10-12$ for EAE mice, $N=4$ for vehicle control mice. Related to Figure 7A. (C) Analysis of disease in the mice that received vehicle controls through FFPE-processed spinal cords undergoing H\&E or LFB staining. Images represent a mouse of the mean disease score of each condition. $\mathrm{N}=4$ for vehicle control mice. Related to Figure 7B. 